LPTENS 99/38

CPTH-S743.1099

NSF-ITP-99-127

$\mathrm{DFF} / 347 / 10 / 99$

LPT-ORSAY 99/80

ROM2F-99/40

hep-th/9911081

\title{
Type I vacua with brane supersymmetry breaking
}

\author{
C. Angelantonj ${ }^{a, b}$, I. Antoniadis ${ }^{b, c}$, G. D'Appollonio ${ }^{d}$, \\ E. Dudas ${ }^{e}$ and A. Sagnotti ${ }^{f}$ \\ ${ }^{a}$ Laboratoire de Physique Théorique de l'École Normale Supérieure ${ }^{\dagger}$ \\ 24, rue Lhomond, F-75231 Paris CEDEX 05 \\ ${ }^{b}$ Centre de Physique Théorique ${ }^{\ddagger}$, École Polytechnique, F-91128 Palaiseau \\ ${ }^{c}$ ITP - Kohn Hall, Univ. of California, Santa Barbara, CA 93106-4030 \\ ${ }^{d}$ Dipartimento di Fisica, Univ. Firenze e INFN, Sez. di Firenze \\ Largo Enrico Fermi 2, I-50125 Firenze \\ e LPT $^{\star}$, Bât. 210, Univ. Paris-Sud, F-91405 Orsay \\ ${ }^{f}$ Dip. di Fisica, Univ. Roma "Tor Vergata" e INFN, Sez. di Roma 2 \\ Via della Ricerca Scientifica 1, I-00133 Roma
}

\begin{abstract}
We show how chiral type I models whose tadpole conditions have no supersymmetric solution can be consistently defined introducing antibranes with non-supersymmetric world volumes. At tree level, the resulting stable non-BPS configurations correspond to tachyon-free spectra, where supersymmetry is broken at the string scale on some (anti)branes but is exact in the bulk, and can be further deformed by the addition of brane-antibrane pairs of the same type. As a result, a scalar potential is generated, that can stabilize some radii of the compact space. This setting has the novel virtue of linking supersymmetry breaking to the consistency requirements of an underlying fundamental theory.
\end{abstract}

${ }^{\dagger}$ Unité mixte du CNRS et de l'ENS, UMR 8549.

‡Unité mixte du CNRS et de l'EP, UMR 7644.

*Unité mixte du CNRS, UMR 8627.

April 14, 2018 


\section{Introduction}

Type I models have become the subject of an intense activity during the last few years, since their perturbative definition [1] offers interesting new possibilities for low-energy phenomenology, and in particular leaves some freedom to lower the string scale well below the Planck mass, if some extra dimensions are large [2, 3, 4, 5]. Their consistency and a number of their most amusing features may be traced to the relation to suitable "parent" models of oriented closed strings, from which their spectra can be derived. In this procedure, a special role is played by "tadpole conditions" for Ramond-Ramond (RR) states [6]. These may be regarded as global neutrality conditions for RR charges [7], constrain the (integer) Chan-Paton multiplicities, and are usually linked to gauge and gravitational anomalies.

The explicit study of type I vacua, however, has revealed an unexpected difficulty: in some interesting chiral four-dimensional models it is apparently impossible to satisfy some of the tadpole conditions [8, 9]. This peculiar phenomenon can often be traced to sign flips of some crosscap contributions or, in more suggestive space-time language, to the reversal of some orientifold charges. Examples are actually known where the solution would lead to negative Chan-Paton multiplicities, thus violating the positivity of the annulus amplitude, or where no solution can be found in general, because crosscaps and boundaries scale differently with the internal volume.

In a recent work, it was shown how this difficulty can be evaded, in a prototype sixdimensional $Z_{2}$ model and in the four-dimensional $Z_{2} \times Z_{2}$ orientifold, if one relaxes the condition that the brane configuration be supersymmetric, thus allowing for vacua including both branes and antibranes. As a result, supersymmetry is broken on some collection of branes at the string scale, while it is preserved (to lowest order) in the bulk and possibly on other branes. Aside from its interest for the consistent definition of type I models, this scenario, termed in [10] "brane supersymmetry breaking", has clearly some beauty of its own if our non-supersymmetric universe is modeled as a brane in a bath of higher- 
dimensional supergravity. Rather than being introduced as a possible deformation, the breaking of supersymmetry in our low-energy world would then be seen as a neat consequence of the internal consistency of the underlying String Theory. Moreover, in this context the present experimental limits on small-distance deviations from Newtonian gravity leave open the exciting possibility that supersymmetry, broken on our world brane, be almost exact a millimeter away from it. We would like to stress that this mechanism links supersymmetry breaking to the string scale, rather than to geometric scales of the internal space, as Scherk-Schwarz 11, 12, 13, 14 or magnetic [15 deformations. The natural distinction between these two settings, however, is closely linked to a geometric interpretation of the two-dimensional Conformal Field Theory, and is somewhat blurred in non-geometric settings, such as those recently explored in [16].

Actually, all these vacua can be further deformed. In particular, in type I models one can add arbitrary numbers of D9 - D $\overline{9}$ and D5 - D $\overline{5}$ brane pairs without violating any RR tadpole condition [17, 18], but introducing additional breakings of supersymmetry on the branes. For instance, starting from the $Z_{3}$ orientifold of [19], one can thus build semi-realistic three-generation models [20]. We would like to stress that the introduction of (anti)5-branes requires in general that additional $Z_{2}$ projections act on the boundaries consistently with the closed spectrum. In this case the resulting configurations are generally unstable, due to the presence of tachyonic modes that, however, can often be lifted by suitable Wilson lines (or, equivalently, by suitable brane displacements). This should be contrasted with the original setting for brane supersymmetry breaking [10], where the brane configuration involves branes and antibranes of different types and is by construction stable. Still, in both settings the models include non-BPS (anti)brane systems of the type considered in [21], whose interactions with gravity are consistently described by type I string theory.

In this work we discuss in more detail the open descendants of two typical examples 
of type IIB orbifolds, where the problem of unsolvable tadpoles was first encountered. We begin in Section 2 with a detailed discussion of four-dimensional $Z_{2} \times Z_{2}$ models with discrete torsion, showing how the unavoidable reversal of some of the orientifold charges is naturally accompanied, in a fully consistent construction, by the simultaneous presence of branes and antibranes. We also discuss similar modifications of the open descendants of the corresponding freely-acting orbifolds recently studied in [14]. In Section 3 we turn to the $T^{6} / Z_{4}$ orbifold, a canonical case where the tadpole conditions can not be solved. Here we can display a consistent non-supersymmetric solution, provided the Klein-bottle projection is also modified, with the net result of lifting the offending twisted tadpole. We should stress, however, that the difficulties met in these two classes of orbifolds are rather distinct. In type-I models, the twisted tadpoles are generally related to non-abelian gauge anomalies, whereas the untwisted ones are related to gravitational anomalies, absent in four dimensions. In the $Z_{2} \times Z_{2}$ models with discrete torsion the twisted tadpoles are actually solvable, whereas the untwisted ones are not, and as a result even the naive supersymmetric open spectrum is free of non-abelian gauge anomalies [8]. On the other hand, in the $Z_{4}$ model with standard Klein bottle the problem is related to the twisted tadpoles, and as a result the naive supersymmetric open spectrum has non-abelian gauge anomalies [9]. In Section 4 we discuss the possible deformations of type I models to stable vacuum configurations including both branes and antibranes of the same type. In particular, we discuss deformations of six-dimensional toroidal compactifications, that may thus include arbitrary pairs of D9 - D $\overline{9}$ and D5 - D $\overline{5}$ branes, and present a similar generalization of the four-dimensional $T^{6} / Z_{4}$ orientifold studied in Section 3. We show explicitly how the presence of additional brane-antibrane pairs, via the resulting NS-NS (Neveu-Schwarz) tadpoles, can actually stabilize the radii of the compact internal space. All the models presented in this paper are meant to provide new instances of the phenomenon discussed in 10]: to lowest order, the D-branes are supersymmetric (with suitable diagonal subgroups of the antibrane gauge groups realized as global symmetries), while the antibranes are not. 
Finally, Section 5 contains our Conclusions and the two Appendices collect some relevant properties of the characters used in the text.

\section{2. $Z_{2} \times Z_{2}$ orientifolds and discrete torsion}

Let us begin by displaying the torus amplitude for the parent type IIB $Z_{2} \times Z_{2}$ orbifold. Aside from the identity, that we denote by $o$, the other three elements act on the three internal two-tori as

$$
g:(+,-,-) \quad, \quad f:(-,+,-) \quad, \quad h:(-,-,+)
$$

In the $Z_{2} \times Z_{2}$ orbifold, one has the freedom of introducing discrete torsion [22, that corresponds to associating a sign $\epsilon= \pm 1$ to the independent modular orbit containing all terms that are twisted and projected by two different orbifold operations. Omitting for brevity the contributions of the space-time bosons, the torus amplitudes of the two $Z_{2} \times Z_{2}$ models are

$$
\begin{aligned}
\mathcal{T} & =\frac{1}{4}\left\{\left|T_{o o}\right|^{2} \Lambda_{1} \Lambda_{2} \Lambda_{3}+\left|T_{o g}\right|^{2} \Lambda_{1}\left|\frac{4 \eta^{2}}{\vartheta_{2}^{2}}\right|^{2}+\left|T_{o f}\right|^{2} \Lambda_{2}\left|\frac{4 \eta^{2}}{\vartheta_{2}^{2}}\right|^{2}+\left|T_{o h}\right|^{2} \Lambda_{3}\left|\frac{4 \eta^{2}}{\vartheta_{2}^{2}}\right|^{2}\right. \\
& +\left|T_{g o}\right|^{2} \Lambda_{1}\left|\frac{4 \eta^{2}}{\vartheta_{4}^{2}}\right|^{2}+\left|T_{g g}\right|^{2} \Lambda_{1}\left|\frac{4 \eta^{2}}{\vartheta_{3}^{2}}\right|^{2}+\left|T_{f o}\right|^{2} \Lambda_{2}\left|\frac{4 \eta^{2}}{\vartheta_{4}^{2}}\right|^{2}+\left|T_{f f}\right|^{2} \Lambda_{2}\left|\frac{4 \eta^{2}}{\vartheta_{3}^{2}}\right|^{2} \\
& +\left|T_{h o}\right|^{2} \Lambda_{3}\left|\frac{4 \eta^{2}}{\vartheta_{4}^{2}}\right|^{2}+\left|T_{h h}\right|^{2} \Lambda_{3}\left|\frac{4 \eta^{2}}{\vartheta_{3}^{2}}\right|^{2} \\
& \left.+\epsilon\left(\left|T_{g h}\right|^{2}+\left|T_{g f}\right|^{2}+\left|T_{f g}\right|^{2}+\left|T_{f h}\right|^{2}+\left|T_{h g}\right|^{2}+\left|T_{h f}\right|^{2}\right)\left|\frac{8 \eta^{3}}{\vartheta_{2} \vartheta_{3} \vartheta_{4}}\right|^{2}\right\}
\end{aligned}
$$

where the $\Lambda_{k}$ are lattice sums for the three internal tori and, throughout the paper, we let $\alpha^{\prime}=2$. We have expressed the torus amplitude in terms of the 16 quantities $(k=o, g, h, f)$

$$
\begin{aligned}
& T_{k o}=\tau_{k o}+\tau_{k g}+\tau_{k h}+\tau_{k f} \quad, \quad T_{k g}=\tau_{k o}+\tau_{k g}-\tau_{k h}-\tau_{k f}, \\
& T_{k h}=\tau_{k o}-\tau_{k g}+\tau_{k h}-\tau_{k f} \quad, \quad T_{k f}=\tau_{k o}-\tau_{k g}-\tau_{k h}+\tau_{k f}
\end{aligned}
$$


where the $16 Z_{2} \times Z_{2}$ characters $\tau_{k l}$ [8], combinations of products of level-one $\mathrm{SO}(2)$ characters, are displayed in Appendix A. The choices $\epsilon=\mp 1$ identify the models with and without discrete torsion, whose low-energy spectra are quite different: in the first $\mathcal{N}=2$ supergravity is coupled to 52 hypermultiplets and 3 vector multiplets, while in the second it is coupled to 4 hypermultiplets and 51 vector multiplets. These spectra correspond to orbifold limits of Calabi-Yau manifolds with Hodge numbers $(51,3)$ and $(3,51)$, respectively.

The $\Omega$ projections that we are considering are implemented by the Klein-bottle amplitudes

$$
\begin{aligned}
\mathcal{K} & =\frac{1}{8}\left\{\left(P_{1} P_{2} P_{3}+P_{1} W_{2} W_{3}+W_{1} P_{2} W_{3}+W_{1} W_{2} P_{3}\right) T_{o o}\right. \\
& \left.+2 \times 16\left[\epsilon_{1}\left(P_{1}+\epsilon W_{1}\right) T_{g o}+\epsilon_{2}\left(P_{2}+\epsilon W_{2}\right) T_{f o}+\epsilon_{3}\left(P_{3}+\epsilon W_{3}\right) T_{h o}\right]\left(\frac{\eta}{\vartheta_{4}}\right)^{2}\right\},
\end{aligned}
$$

where $P_{k}$ and $W_{k}$ denote the restrictions of the lattice sums $\Lambda_{k}$ to their momentum and winding sublattices. Discrete torsion has a neat effect [14] on $\left(P_{k}+\epsilon W_{k}\right)$ : if $\epsilon=1$, the massless twisted contributions are diagonal combinations of the $\tau_{k l}$, and appear in the Klein bottle, while if $\epsilon=-1$ they are off-diagonal combinations, and do not contribute to it. Consistently with the crosscap constraint [23], (2.4) can accommodate three additional signs $\epsilon_{k}$. Actually, these are not independent, but are linked to the parameter $\epsilon$ by the constraint

$$
\epsilon_{1} \epsilon_{2} \epsilon_{3}=\epsilon
$$

One can write this amplitude as

$$
\mathcal{K}=\frac{1}{8}\left\{\left(P_{1} P_{2} P_{3}+\frac{1}{2} P_{k} W_{l} W_{m}\right) T_{o o}+2 \times 16 \epsilon_{k}\left(P_{k}+\epsilon W_{k}\right) T_{k o}\left(\frac{\eta}{\vartheta_{4}}\right)^{2}\right\}
$$

where we have resorted to a compact notation, used extensively in the following: summations over repeated indices and symmetrizations over distinct indices are left implicit. An $S$ transformation turns this expression into the corresponding vacuum-channel amplitude

$$
\tilde{\mathcal{K}}=\frac{2^{5}}{8}\left\{\left(v_{1} v_{2} v_{3} W_{1}^{e} W_{2}^{e} W_{3}^{e}+\frac{v_{k}}{2 v_{l} v_{m}} W_{k}^{e} P_{l}^{e} P_{m}^{e}\right) T_{o o}+2 \epsilon_{k}\left(v_{k} W_{k}^{e}+\epsilon \frac{P_{k}^{e}}{v_{k}}\right) T_{o k}\left(\frac{2 \eta}{\vartheta_{2}}\right)^{2}\right\}
$$


where the superscript $e$ denotes the restriction of the lattice sums to their even terms and the $v_{k}$ denote the volumes of the three internal tori. At the origin of the lattices, the constraint (2.5) leads to an expression whose coefficients are perfect squares,

$$
\begin{aligned}
\tilde{\mathcal{K}}_{0}= & \frac{2^{5}}{8}\left\{\left(\sqrt{v_{1} v_{2} v_{3}}+\epsilon_{1} \sqrt{\frac{v_{1}}{v_{2} v_{3}}}+\epsilon_{2} \sqrt{\frac{v_{2}}{v_{1} v_{3}}}+\epsilon_{3} \sqrt{\frac{v_{3}}{v_{1} v_{2}}}\right)^{2} \tau_{o o}\right. \\
& +\left(\sqrt{v_{1} v_{2} v_{3}}+\epsilon_{1} \sqrt{\frac{v_{1}}{v_{2} v_{3}}}-\epsilon_{2} \sqrt{\frac{v_{2}}{v_{1} v_{3}}}-\epsilon_{3} \sqrt{\frac{v_{3}}{v_{1} v_{2}}}\right)^{2} \tau_{o g} \\
& +\left(\sqrt{v_{1} v_{2} v_{3}}-\epsilon_{1} \sqrt{\frac{v_{1}}{v_{2} v_{3}}}+\epsilon_{2} \sqrt{\frac{v_{2}}{v_{1} v_{3}}}-\epsilon_{3} \sqrt{\frac{v_{3}}{v_{1} v_{2}}}\right)^{2} \tau_{o f} \\
& \left.+\left(\sqrt{v_{1} v_{2} v_{3}}-\epsilon_{1} \sqrt{\frac{v_{1}}{v_{2} v_{3}}}-\epsilon_{2} \sqrt{\frac{v_{2}}{v_{1} v_{3}}}+\epsilon_{3} \sqrt{\frac{v_{3}}{v_{1} v_{2}}}\right)^{2} \tau_{o h}\right\},
\end{aligned}
$$

that shows rather neatly how the choice $\epsilon_{k}=-1$ reverts the charge of the $05_{k}$ orientifold plane. While manifestly compatible with the usual positivity requirements, this reversal clearly affects the tadpole conditions, that require the introduction of antibranes. In this respect, it should be appreciated that, according to (2.5), discrete torsion implies the reversal of at least one of the 05 charges. Therefore, taking into account the presence of the $\epsilon_{k}$, one can identify four classes of models, determined by the independent choices for $\left(\epsilon_{1}, \epsilon_{2}, \epsilon_{3}\right)$. If $\epsilon=1$, the choice $(+,+,+)$ gives the model discussed in [8, 24], with 48 chiral multiplets from the closed twisted sectors, while the choice $(+,-,-)$ gives a model with 16 chiral multiplets and 32 vector multiplets from the twisted sectors. On the other hand, for $\epsilon=-1$ the two choices $(+,+,-)$ and $(-,-,-)$ yield the same massless twisted spectrum, namely 48 chiral multiplets.

In order to describe the annulus amplitude, it is convenient to introduce a compact notation. If $T_{k l}^{\mathrm{NS}}\left(T_{k l}^{\mathrm{R}}\right)$ denote the NS (R) parts of the usual combinations of supersymmetric $Z_{2} \times Z_{2}$ characters, we thus define

$$
\tilde{T}_{k l}^{(\varepsilon)}=T_{k l}^{\mathrm{NS}}-\varepsilon T_{k l}^{\mathrm{R}}
$$

where $\varepsilon= \pm 1$. Whereas $\tilde{T}_{k l}^{(+)}\left(=T_{k l}^{(+)}\right)$, that in the following we simply denote by $T_{k l}$ for the sake of brevity, form a closed set under $S$ modular transformations, the additional 
combinations $\tilde{T}_{k l}^{(-)}$with reversed $\mathrm{RR}$ charges, associated to the interactions between branes and antibranes, do not. As a result, the corresponding terms in $\mathcal{A}$, describing open strings stretched between branes and antibranes, contain new combinations $T_{k l}^{(-)}$, obtained from the $T_{k l}^{(+)}$interchanging $O_{2}$ with $V_{2}$ and $S_{2}$ with $C_{2}$ in the last three factors, as explained in Appendix A.

The transverse-channel annulus amplitude is

$$
\begin{aligned}
\tilde{\mathcal{A}} & =\frac{2^{-5}}{8}\left\{\left(N_{o}^{2} v_{1} v_{2} v_{3} W_{1} W_{2} W_{3}+\frac{D_{k ; o}^{2} v_{k}}{2 v_{l} v_{m}} W_{k} P_{l} P_{m}\right) T_{o o}\right. \\
& +4\left[\left(N_{k}^{2}+D_{k ; k}^{2}\right) v_{k} W_{k}+D_{l \neq k ; k}^{2} \frac{P_{k}}{v_{k}}\right] T_{k o}\left(\frac{2 \eta}{\vartheta_{4}}\right)^{2} \\
& +2 N_{o} D_{k ; o} v_{k} W_{k} \tilde{T}_{o k}^{\left(\epsilon_{k}\right)}\left(\frac{2 \eta}{\vartheta_{2}}\right)^{2}+2 N_{k} D_{k ; k} v_{k} W_{k} \tilde{T}_{k k}^{\left(\epsilon_{k}\right)}\left(\frac{2 \eta}{\vartheta_{3}}\right)^{2}+4 N_{l} D_{k \neq l ; l} \tilde{T}_{l k}^{\left(\epsilon_{k}\right)} \frac{8 \eta^{3}}{\vartheta_{2} \vartheta_{3} \vartheta_{4}} \\
& \left.+D_{k ; o} D_{l ; o} \frac{P_{m}}{v_{m}} \tilde{T}_{o m}^{\left(\epsilon_{k} \epsilon_{l}\right)}\left(\frac{2 \eta}{\vartheta_{2}}\right)^{2}+D_{k ; m} D_{l ; m} \frac{P_{m}}{v_{m}} \tilde{T}_{m m}^{\left(\epsilon_{k} \epsilon_{l}\right)}\left(\frac{2 \eta}{\vartheta_{3}}\right)^{2}+4 D_{k ; k} D_{l ; k} \tilde{T}_{k m}^{\left(\epsilon_{k} \epsilon_{l}\right)} \frac{8 \eta^{3}}{\vartheta_{2} \vartheta_{3} \vartheta_{4}}\right\}
\end{aligned}
$$

where $N_{o}, D_{g ; o}, D_{f ; o}$ and $D_{h ; o}$ are the charges for the D9 branes and for the three sets of D5 or D $\overline{5}$ branes wrapped around the first, second and third torus. In a similar fashion, $N_{k}$, $D_{g ; k}, D_{f ; k}$ and $D_{h ; k}(k=g, f, h)$ parametrize the breakings induced by the three orbifold operations $g, f$ and $h$. As expected, the RR part of every term describing the interaction between a brane and an antibrane has a reversed sign. The untwisted terms at the origin of the lattice sums rearrange themselves into perfect squares:

$$
\begin{aligned}
\tilde{\mathcal{A}}_{0}= & \frac{2^{-5}}{8}\left\{\left(N_{o} \sqrt{v_{1} v_{2} v_{3}}+D_{g ; o} \sqrt{\frac{v_{1}}{v_{2} v_{3}}}+D_{f ; o} \sqrt{\frac{v_{2}}{v_{1} v_{3}}}+D_{h ; o} \sqrt{\frac{v_{3}}{v_{1} v_{2}}}\right)^{2} \tau_{o o}^{\mathrm{NS}}\right. \\
& -\left(N_{o} \sqrt{v_{1} v_{2} v_{3}}+\epsilon_{1} D_{g ; o} \sqrt{\frac{v_{1}}{v_{2} v_{3}}}+\epsilon_{2} D_{f ; o} \sqrt{\frac{v_{2}}{v_{1} v_{3}}}+\epsilon_{3} D_{h, o} \sqrt{\frac{v_{3}}{v_{1} v_{2}}}\right)^{2} \tau_{o o}^{\mathrm{R}} \\
& +\left(N_{o} \sqrt{v_{1} v_{2} v_{3}}+D_{g ; o} \sqrt{\frac{v_{1}}{v_{2} v_{3}}}-D_{f ; o} \sqrt{\frac{v_{2}}{v_{1} v_{3}}}-D_{h ; o} \sqrt{\frac{v_{3}}{v_{1} v_{2}}}\right)^{2} \tau_{o g}^{\mathrm{NS}} \\
& -\left(N_{o} \sqrt{v_{1} v_{2} v_{3}}+\epsilon_{1} D_{g ; o} \sqrt{\frac{v_{1}}{v_{2} v_{3}}}-\epsilon_{2} D_{f ; o} \sqrt{\frac{v_{2}}{v_{1} v_{3}}}-\epsilon_{3} D_{h ; o} \sqrt{\frac{v_{3}}{v_{1} v_{2}}}\right)^{2} \tau_{o g}^{\mathrm{R}} \\
& +\left(N_{o} \sqrt{v_{1} v_{2} v_{3}}-D_{g ; o} \sqrt{\frac{v_{1}}{v_{2} v_{3}}}+D_{f ; o} \sqrt{\frac{v_{2}}{v_{1} v_{3}}}-D_{h ; o} \sqrt{\frac{v_{3}}{v_{1} v_{2}}}\right)^{2} \tau_{o f}^{\mathrm{NS}}
\end{aligned}
$$




$$
\begin{aligned}
& -\left(N_{o} \sqrt{v_{1} v_{2} v_{3}}-\epsilon_{1} D_{g ; o} \sqrt{\frac{v_{1}}{v_{2} v_{3}}}+\epsilon_{2} D_{f ; o} \sqrt{\frac{v_{2}}{v_{1} v_{3}}}-\epsilon_{3} D_{h ; o} \sqrt{\frac{v_{3}}{v_{1} v_{2}}}\right)^{2} \tau_{o f}^{\mathrm{R}} \\
& +\left(N_{o} \sqrt{v_{1} v_{2} v_{3}}-D_{g ; o} \sqrt{\frac{v_{1}}{v_{2} v_{3}}}-D_{f ; o} \sqrt{\frac{v_{2}}{v_{1} v_{3}}}+D_{h ; o} \sqrt{\frac{v_{3}}{v_{1} v_{2}}}\right)^{2} \tau_{o h}^{\mathrm{NS}} \\
& \left.-\left(N_{o} \sqrt{v_{1} v_{2} v_{3}}-\epsilon_{1} D_{g ; o} \sqrt{\frac{v_{1}}{v_{2} v_{3}}}-\epsilon_{2} D_{f ; o} \sqrt{\frac{v_{2}}{v_{1} v_{3}}}+\epsilon_{3} D_{h ; o} \sqrt{\frac{v_{3}}{v_{1} v_{2}}}\right)^{2} \tau_{o h}^{\mathrm{R}}\right\} .
\end{aligned}
$$

Moreover, the breaking terms reflect rather neatly the geometry of the (anti)brane configuration. Indeed, the coefficient that multiplies a given twisted character is a sum of squares associated to the fixed tori of the various twisted sectors, and each square contains the breaking terms for the branes present in the fixed tori, with factors $\sqrt{v}$ if they are wrapped around them or $1 / \sqrt{v}$ if they are localized on them. The relative coefficients of these terms are also directly linked to the brane geometry, and are given by

$$
\sqrt{\frac{\# \text { of fixed tori }}{\# \text { of occupied fixed tori }}} .
$$

Thus, for a given twisted sector, the numerator counts the fixed tori, while the denominator counts the fixed tori where branes are actually present. Moreover, the $\mathrm{R}$ portions of the characters describing brane-antibrane exchanges have reverted signs also in these twisted contributions, as expected. For instance, in the $g$-twisted sector of the $(++-)$ model, that contains $\mathrm{D} \overline{5}_{3}$ branes, the reflection coefficients for the massless modes in $\tau_{g h}$ are

$$
\begin{aligned}
& \frac{2^{-5}}{8}\left[\left(N_{g} \sqrt{v_{1}}-4 D_{g ; g} \sqrt{v_{1}}-2 D_{f ; g} \frac{1}{\sqrt{v_{1}}}+2 D_{h ; g} \frac{1}{\sqrt{v_{1}}}\right)^{2}+3\left(N_{g} \sqrt{v_{1}}-2 D_{f ; g} \frac{1}{\sqrt{v_{1}}}\right)^{2}\right. \\
& \left.\quad+3\left(N_{g} \sqrt{v_{1}}+2 D_{h ; g} \frac{1}{\sqrt{v_{1}}}\right)^{2}+9 N_{g}^{2} v_{1}\right]
\end{aligned}
$$

for the NS portion, and

$$
\begin{aligned}
& \frac{2^{-5}}{8}\left[\left(N_{g} \sqrt{v_{1}}-4 D_{g ; g} \sqrt{v_{1}}-2 D_{f ; g} \frac{1}{\sqrt{v_{1}}}-2 D_{h ; g} \frac{1}{\sqrt{v_{1}}}\right)^{2}+3\left(N_{g} \sqrt{v_{1}}-2 D_{f ; g} \frac{1}{\sqrt{v_{1}}}\right)^{2}\right. \\
& \left.\quad+3\left(N_{g} \sqrt{v_{1}}-2 D_{h ; g} \frac{1}{\sqrt{v_{1}}}\right)^{2}+9 N_{g}^{2} v_{1}\right]
\end{aligned}
$$

for the R portion. According to (2.12), the coefficient of $N_{g}$ is $\sqrt{v_{1}}$, since the D9 are wrapped around all fixed tori, the coefficient of $D_{g ; g}$ is $4 \sqrt{v_{1}}$, since the D $5_{1}$ are only wrapped around 
one fixed torus, while the coefficients of $D_{f ; g}$ and $D_{h ; g}$ are $2 / \sqrt{v_{1}}$, since the $\mathrm{D} 5_{2}$ and $\mathrm{D} \overline{5}_{3}$ are confined to four of the fixed tori. Finally, out of the $16 \mathrm{~g}$-fixed tori, one sees all the branes, three see only the D9 and the D $5_{2}$, three see only the D9 and the $\mathrm{D} \overline{5}_{3}$ and, finally, nine see only the D9.

The direct-channel annulus amplitude is then

$$
\begin{aligned}
\mathcal{A} & =\frac{1}{8}\left\{\left(N_{o}^{2} P_{1} P_{2} P_{3}+\frac{D_{k ; o}^{2}}{2} P_{k} W_{l} W_{m}\right) T_{o o}+\left[\left(N_{k}^{2}+D_{k ; k}^{2}\right) P_{k}+D_{l \neq k ; k}^{2} W_{k}\right] T_{o k}\left(\frac{2 \eta}{\vartheta_{2}}\right)^{2}\right. \\
& +2 N_{o} D_{k ; o} P_{k} T_{k o}^{\left(\epsilon_{k}\right)}\left(\frac{\eta}{\vartheta_{4}}\right)^{2}-2 N_{k} D_{k ; k} P_{k} T_{k k}^{\left(\epsilon_{k}\right)}\left(\frac{\eta}{\vartheta_{3}}\right)^{2} \\
& +2 i(-1)^{k+l} N_{l} D_{k \neq l ; l} T_{k l}^{\left(\epsilon_{k}\right)} \frac{2 \eta^{3}}{\vartheta_{2} \vartheta_{3} \vartheta_{4}}+D_{k ; o} D_{l ; o} W_{m} T_{m o}^{\left(\epsilon_{k} \epsilon_{l}\right)}\left(\frac{\eta}{\vartheta_{4}}\right)^{2} \\
& \left.-D_{k ; m} D_{l ; m} W_{m} T_{m m}^{\left(\epsilon_{k} \epsilon_{l}\right)}\left(\frac{\eta}{\vartheta_{3}}\right)^{2}+2 i(-1)^{m+k} D_{k ; k} D_{l ; k} T_{m k}^{\left(\epsilon_{k} \epsilon_{l}\right)} \frac{2 \eta^{3}}{\vartheta_{2} \vartheta_{3} \vartheta_{4}}\right\}
\end{aligned}
$$

where in the signs $(-1)^{k+l}$ and $(-1)^{m+k} k, l, m$ take the values $1,2,3$ for the $g, f$, and $h$ generators. The transverse-channel amplitudes $\tilde{\mathcal{K}}$ and $\tilde{\mathcal{A}}$ determine by standard methods the transverse Möbius amplitude

$$
\begin{aligned}
\tilde{\mathcal{M}} & =-\frac{1}{4}\left\{N_{o} v_{1} v_{2} v_{3} W_{1}^{e} W_{2}^{e} W_{3}^{e} \hat{T}_{o o}+N_{o} v_{k} W_{k}^{e} \epsilon_{k} \hat{T}_{o k}\left(\frac{2 \hat{\eta}}{\hat{\vartheta}_{2}}\right)^{2}+\frac{v_{k}}{2 v_{l} v_{m}} D_{k ; o} W_{k}^{e} P_{l}^{e} P_{m}^{e} \epsilon_{k} \hat{\tilde{T}}_{o o}^{\left(\epsilon_{k}\right)}\right. \\
& \left.+\left(D_{l ; o} \epsilon_{k} \frac{P_{m}^{e}}{v_{m}} \hat{\tilde{T}}_{o m}^{\left(\epsilon_{l}\right)}+D_{k ; o} v_{k} W_{k}^{e} \hat{\tilde{T}}_{o k}^{\left(\epsilon_{k}\right)}\right)\left(\frac{2 \hat{\eta}}{\hat{\vartheta}_{2}}\right)^{2}\right\}
\end{aligned}
$$

and, after a $P$ transformation, the direct-channel Möbius amplitude

$$
\begin{aligned}
\mathcal{M} & =-\frac{1}{8}\left\{N_{o} P_{1} P_{2} P_{3} \hat{T}_{o o}-N_{o} P_{k} \epsilon_{k} \hat{T}_{o k}\left(\frac{2 \hat{\eta}}{\hat{\vartheta}_{2}}\right)^{2}+\frac{1}{2} D_{k ; o} P_{k} W_{l} W_{m} \epsilon_{k} \hat{\tilde{T}}_{o o}^{\left(\epsilon_{k}\right)}\right. \\
& \left.-\left(D_{l ; o} \epsilon_{k} W_{m} \hat{\tilde{T}}_{o m}^{\left(\epsilon_{l}\right)}+D_{k ; o} P_{k} \hat{\tilde{T}}_{o k}^{\left(\epsilon_{k}\right)}\right)\left(\frac{2 \hat{\eta}}{\hat{\vartheta}_{2}}\right)^{2}\right\} .
\end{aligned}
$$

From the transverse amplitudes one can now read the tadpole conditions

$$
\begin{array}{cl}
N_{o}=32, & N_{g}=N_{f}=N_{h}=0, \\
D_{k ; o}=32, & D_{k ; g}=D_{k ; f}=D_{k ; h}=0 .
\end{array}
$$




\subsection{Massless Spectra}

The models where only one $\epsilon_{k}$ is negative have discrete torsion and contain one D $\overline{5}$. For the D9 and the two sets of D5 branes, the gauge groups are $\mathrm{U}(8) \times \mathrm{U}(8)$, with $\mathcal{N}=1$ supersymmetry, while for the $\bar{D} \overline{5}$ branes the gauge group is $\operatorname{USp}(8)^{4}$, with $\mathcal{N}=0$. Moreover, the 59 and $5_{k} 5_{l}$ strings are supersymmetric, while the $9 \overline{5}$ and $5_{k} \overline{5}$ strings are not. Let us discuss in some detail the case $\left(\epsilon_{1}, \epsilon_{2}, \epsilon_{3}\right)=(++-)$, that contains D $\overline{5}$ branes wrapped around the third torus. To this end, let us parametrize the charges as

$$
\begin{aligned}
N_{o} & =o+g+\bar{o}+\bar{g}, & N_{g} & =i(o+g-\bar{o}-\bar{g}), \\
N_{f} & =i(o-g-\bar{o}+\bar{g}), & N_{h} & =o-g+\bar{o}-\bar{g}, \\
D_{g ; o} & =o_{1}+g_{1}+\bar{o}_{1}+\bar{g}_{1}, & D_{g ; g} & =i\left(o_{1}+g_{1}-\bar{o}_{1}-\bar{g}_{1}\right), \\
D_{g ; f} & =o_{1}-g_{1}+\bar{o}_{1}-\bar{g}_{1}, & D_{g ; h} & =-i\left(o_{1}-g_{1}-\bar{o}_{1}+\bar{g}_{1}\right), \\
D_{f ; o} & =o_{2}+g_{2}+\bar{o}_{2}+\bar{g}_{2}, & D_{f ; g} & =o_{2}-g_{2}+\bar{o}_{2}-\bar{g}_{2}, \\
D_{f ; f} & =i\left(o_{2}+g_{2}-\bar{o}_{2}-\bar{g}_{2}\right), & D_{f ; h} & =i\left(o_{2}-g_{2}-\bar{o}_{2}+\bar{g}_{2}\right), \\
D_{h ; o} & =a+b+c+d, & D_{h ; g} & =a+b-c-d, \\
D_{h ; f} & =a-b+c-d, & D_{h ; h} & =a-b-c+d,
\end{aligned}
$$

and extract the massless spectrum from the amplitudes at the origin of the lattices. The $99,5_{1} 5_{1}$ and $5_{2} 5_{2}$ sectors have $\mathcal{N}=1$ supersymmetry, and all give gauge groups $\mathrm{U}(8) \times$ $\mathrm{U}(8)$, with chiral multiplets in the representations $(8,8),(8, \overline{8}),(28,1),(1,28)$ and their

conjugates. Moreover, as expected, the $95_{1}, 95_{2}$ and $5_{1} 5_{2}$ strings are also supersymmetric, and contain chiral multiplets in the representations

$$
\begin{aligned}
95_{1}: & (8,1 ; 1, \overline{8}),(1,8 ; \overline{8}, 1),(\overline{8}, 1 ; 8,1),(1, \overline{8} ; 1,8), \\
95_{2}: & (8,1 ; 1, \overline{8}),(1, \overline{8} ; \overline{8}, 1),(\overline{8}, 1 ; 8,1),(1,8 ; 1,8), \\
55_{1}: & (8,1 ; 8,1),(1,8 ; \overline{8}, 1),(\overline{8}, 1 ; 1,8),(1, \overline{8} ; 1, \overline{8}) .
\end{aligned}
$$


On the other hand, the strings whose ends live on the antibrane give rise to supersymmetric spectra, even if the annulus contains supersymmetric characters, since bosons and fermions are treated differently by $\mathcal{M}$. Thus, the $\overline{5}_{3} \overline{5}_{3}$ sector contributes a gauge group $\mathrm{USp}(8)^{4}$, with Weyl spinors in the $(28,1,1,1)$ and in three additional permutations, and chiral multiplets in the $(8,8,1,1)$ and in five additional permutations. Finally, the strings stretched between a brane and an antibrane have non-supersymmetric spectra, with Weyl spinors and complex scalars in the representations

$$
\begin{aligned}
& 9 \overline{5}_{3} \text { spinors : }(\overline{8}, 1 ; 8,1,1,1),(1, \overline{8} ; 1,8,1,1),(1,8 ; 1,1,8,1), \quad(8,1 ; 1,1,1,8) \\
& \text { scalars : }(\overline{8}, 1 ; 1,8,1,1),(1, \overline{8} ; 8,1,1,1), \quad(1,8 ; 1,1,1,8), \quad(8,1 ; 1,1,8,1) \text {, } \\
& 5_{1} \overline{5}_{3} \text { spinors : }(\overline{8}, 1 ; 1,1,8,1), \quad(1, \overline{8} ; 1,1,1,8), \quad(1,8 ; 1,8,1,1), \quad(8,1 ; 8,1,1,1) \\
& \text { scalars : }(\overline{8}, 1 ; 1,1,1,8),(1, \overline{8} ; 1,1,8,1),(1,8 ; 8,1,1,1), \quad(8,1 ; 1,8,1,1) \text {, } \\
& 5_{2} \overline{5}_{3} \text { spinors : }(8,1 ; 8,1,1,1), \quad(1, \overline{8} ; 1,1,1,8), \quad(1,8 ; 1,1,8,1), \quad(\overline{8}, 1 ; 1,8,1,1) \\
& \text { scalars : }(\overline{8}, 1 ; 1,1,1,8),(1, \overline{8} ; 1,8,1,1),(1,8 ; 8,1,1,1), \quad(8,1 ; 1,1,8,1) \text {. }
\end{aligned}
$$

The choice $\left(\epsilon_{1}, \epsilon_{2}, \epsilon_{3}\right)=(---)$ also corresponds to a model with discrete torsion. In this case, however, there are D9 branes and three sets of D $\overline{5}$ branes, while the charges are to be parametrized as

$$
\begin{aligned}
N_{o} & =a+b+c+d, & N_{g} & =a+b-c-d, \\
N_{f} & =a-b+c-d, & N_{h} & =a-b-c+d, \\
D_{g ; o} & =o_{1}+g_{1}+\bar{o}_{1}+\bar{g}_{1}, & D_{g ; g} & =o_{1}-g_{1}+\bar{o}_{1}-\bar{g}_{1}, \\
D_{g ; f} & =i\left(o_{1}+g_{1}-\bar{o}_{1}-\bar{g}_{1}\right), & D_{g ; h} & =i\left(o_{1}-g_{1}-\bar{o}_{1}+\bar{g}_{1}\right), \\
D_{f ; o} & =o_{2}+g_{2}+\bar{o}_{2}+\bar{g}_{2}, & D_{f ; g} & =i\left(o_{2}+g_{2}-\bar{o}_{2}-\bar{g}_{2}\right), \\
D_{f ; f} & =o_{2}-g_{2}+\bar{o}_{2}-\bar{g}_{2}, & D_{f ; h} & =-i\left(o_{2}-g_{2}-\bar{o}_{2}+\bar{g}_{2}\right), \\
D_{h ; o} & =o_{3}+g_{3}+\bar{o}_{3}+\bar{g}_{3}, & D_{h ; g} & =i\left(o_{3}+g_{3}-\bar{o}_{3}-\bar{g}_{3}\right),
\end{aligned}
$$




$$
D_{h ; f}=i\left(o_{3}-g_{3}-\bar{o}_{3}+\bar{g}_{3}\right), \quad D_{h ; h}=o_{3}-g_{3}+\bar{o}_{3}-\bar{g}_{3} .
$$

The D9 branes have $\mathcal{N}=1$ supersymmetry, with gauge group $\mathrm{SO}(8)^{4}$ and chiral multiplets in the $(8,8,1,1)$ and five permutations. Moreover, each antibrane gives a nonsupersymmetric spectrum, with gauge group $\mathrm{U}(8) \times \mathrm{U}(8)$, chiral multiplets in the $(8,8)$, $(8, \overline{8})$ and in their conjugates, spinors in the $(28,1),(\overline{28}, 1),(1,28),(1, \overline{28})$ and complex scalars in the $(36,1),(\overline{36}, 1),(1,36),(1, \overline{36})$. We would like to stress that in this case the gauginos are massless, since the Möbius amplitude does not affect the adjoint representations of unitary groups. Finally, $\overline{5}_{k} \overline{5}_{l}$ sectors give chiral multiplets in the representations

$$
\begin{array}{ll}
\overline{5}_{1} \overline{5}_{2} & (8,1 ; 8,1),(\overline{8}, 1 ; 1,8),(1,8 ; \overline{8}, 1),(1, \overline{8} ; 1, \overline{8}), \\
\overline{5}_{1} \overline{5}_{3} & (8,1 ; \overline{8}, 1),(\overline{8}, 1 ; 1, \overline{8}),(1,8 ; 1,8),(1, \overline{8} ; 8,1), \\
\overline{5}_{2} \overline{5}_{3} & (8,1 ; \overline{8}, 1),(\overline{8}, 1 ; 1,8),(1,8 ; 1, \overline{8}),(1, \overline{8} ; 8,1),
\end{array}
$$

and the non-supersymmetric $9 \overline{5}_{k}$ sectors contain Weyl spinors and complex scalars in the representations

$$
\begin{aligned}
& 9 \overline{5}_{1} \quad \text { spinors : }(8,1,1,1 ; 8,1),(1,8,1,1 ; \overline{8}, 1),(1,1,8,1 ; 1,8),(1,1,1,8 ; 1, \overline{8}) \text {, } \\
& \text { scalars : } \quad(8,1,1,1 ; 1,8),(1,8,1,1 ; 1, \overline{8}), \quad(1,1,8,1 ; 8,1), \quad(1,1,1,8 ; \overline{8}, 1) \text {, } \\
& 9 \overline{5}_{2} \quad \text { spinors : }(8,1,1,1 ; 8,1),(1,8,1,1 ; 1,8),(1,1,8,1 ; \overline{8}, 1),(1,1,1,8 ; 1, \overline{8}) \text {, } \\
& \text { scalars : }(8,1,1,1 ; 1,8),(1,8,1,1 ; 8,1),(1,1,8,1 ; 1, \overline{8}),(1,1,1,8 ; \overline{8}, 1) \text {, } \\
& 9 \overline{5}_{3} \quad \text { spinors : }(8,1,1,1 ; \overline{8}, 1),(1,8,1,1 ; 1, \overline{8}),(1,1,8,1 ; 1,8),(1,1,1,8 ; 8,1) \text {, } \\
& \text { scalars : } \quad(8,1,1,1 ; 1, \overline{8}),(1,8,1,1 ; \overline{8}, 1), \quad(1,1,8,1 ; 8,1), \quad(1,1,1,8 ; 1,8) \text {. }
\end{aligned}
$$

All chiral spectra thus obtained are free of non-abelian anomalies.

On the other hand, the models without discrete torsion are not chiral. The choice $\left(\epsilon_{1}, \epsilon_{2}, \epsilon_{3}\right)=(+++)$, discussed in [8] and worked out in detail in [24], leads to a gauge group USp $(16)^{4}$. Another model, without discrete torsion but with two D $\overline{5}$ branes, can be 
obtained letting two of the $\epsilon_{k}$ be negative. The D9 and D5 branes give orthogonal gauge groups with $\mathcal{N}=1$ supersymmetry, while the two $\mathrm{D} \overline{5}$ branes give symplectic gauge groups with no supersymmetry. For instance, with the choice $(+--)$

$$
\begin{aligned}
\mathcal{A}_{0} & =\frac{1}{8}\left\{\left(N_{o}^{2}+D_{g ; o}^{2}+D_{f ; o}^{2}+D_{h ; o}^{2}\right) T_{o o}+2 N_{o} D_{g ; o} T_{g o}+2 N_{o} D_{f ; o} T_{f o}^{(-)}+2 N_{o} D_{h ; o} T_{h o}^{(-)}\right. \\
& \left.+2 D_{g ; o} D_{f ; o} T_{h o}^{(-)}+2 D_{g ; o} D_{h ; o} T_{f o}^{(-)}+2 D_{f ; o} D_{h ; o} T_{g o}\right\} \\
\mathcal{M}_{0} & =-\frac{1}{4}\left\{\left(N_{o}+D_{g ; o}\right)\left(\tau_{o o}-\tau_{o g}+\tau_{o f}+\tau_{o h}\right)\right. \\
& \left.-\left(D_{f ; o}+D_{h ; o}\right)\left[\left(\tau_{o o}^{\mathrm{NS}}-\tau_{o g}^{\mathrm{NS}}+\tau_{o f}^{\mathrm{NS}}+\tau_{o h}^{\mathrm{NS}}\right)+\left(\tau_{o o}^{\mathrm{R}}-\tau_{o g}^{\mathrm{R}}+\tau_{o f}^{\mathrm{R}}+\tau_{o h}^{\mathrm{R}}\right)\right]\right\}
\end{aligned}
$$

and there are no breaking terms in the annulus. After a suitable rescaling of the charge multiplicities, the $\overline{\mathrm{D}}_{2}$ and the $\overline{\mathrm{D}}_{3}$ branes give non-supersymmetric spectra, with USp(16) gauge groups, spinors in the 136 and in three copies of the 120 and scalars in the 120 and in two copies of the 136 . The 99 and $5_{1} 5_{1}$ sectors have $\mathcal{N}=1$ supersymmetry, gauge group $\mathrm{SO}(16)$ and chiral multiplets in the 136 and in two copies of the 120. Finally, there are two chiral multiplets in the representation $(16,16)$ arising from the $95_{1}$ and the $\overline{5}_{2} \overline{5}_{3}$ sectors and complex scalars and Weyl spinors in bifundamental representations arising from the $9 \overline{5}_{2}$, $9 \overline{5}_{3}, 5_{1} \overline{5}_{2}$ and $5_{1} \overline{5}_{3}$ sectors.

The fact that the $Z_{2} \times Z_{2}$ orientifold with discrete torsion naturally leads to nonsupersymmetric spectra can also be argued considering F-theory on the $T^{8} / Z_{2} \times Z_{2} \times Z_{2}$ with discrete torsion [25], since the blow-up of this eight-dimensional orbifold is only birational to a Calabi-Yau fourfold 国.

\subsection{Comments on freely acting $Z_{2} \times Z_{2}$ orientifolds with brane supersymmetry breaking}

In all the models discussed in [14], one can introduce D $\overline{5}$ branes simply reverting some signs in the twisted sectors of the Klein bottle, as in the previous case. This procedure

\footnotetext{
${ }^{1}$ We would like to thank Zurab Kakushadze and Koushik Ray for calling this correspondence to our attention.
} 
generates models with branes, antibranes and various amounts of supersymmetry. For instance, the $p_{23}$ model of [14], with $\frac{1}{2}$-momentum shifts along the last two tori, can be turned into a different model, described by

$$
\begin{aligned}
\mathcal{K} & =\frac{1}{8}\left\{T_{o o}\left[P_{1} P_{2} P_{3}+P_{1} W_{2} W_{3}+W_{1}(-1)^{m_{2}} P_{2} W_{3}+W_{1} W_{2}(-1)^{m_{3}} P_{3}\right]\right. \\
& \left.-2 \times 16 T_{g o} P_{1}\left(\frac{\eta}{\vartheta_{4}}\right)^{2}\right\} \\
\mathcal{A} & =\frac{1}{8}\left\{T_{o o}\left[N_{o}^{2} P_{1} P_{2} P_{3}+\frac{D_{g ; o}^{2}}{2} P_{1}\left(W_{2} W_{3}+W_{2}^{n+1 / 2} W_{3}^{n+1 / 2}\right)\right]\right. \\
& +\left(N_{g}^{2}+2 D_{g ; g}^{2}\right) T_{o g} P_{1}\left(\frac{2 \eta}{\vartheta_{2}}\right)^{2}+N_{f}^{2} T_{o f}(-1)^{m_{2}} P_{2}\left(\frac{2 \eta}{\vartheta_{2}}\right)^{2}+N_{h}^{2} T_{o h}(-1)^{m_{3}} P_{3}\left(\frac{2 \eta}{\vartheta_{2}}\right)^{2} \\
& \left.+2 N_{o} D_{g ; o} T_{g o}^{(-)} P_{1}\left(\frac{\eta}{\vartheta_{4}}\right)^{2}-4 N_{g} D_{g ; g} T_{g g}^{(-)} P_{1}\left(\frac{\eta}{\vartheta_{3}}\right)^{2}\right\}, \\
\mathcal{M} & =-\frac{1}{8}\left\{\left(\hat{T}_{o o} N_{o} P_{1} P_{2} P_{3}-\hat{\tilde{T}}_{o o}^{(-)} D_{g ; o} P_{1} W_{2} W_{3}\right)+\left(\hat{T}_{o g} N_{o}-\hat{\tilde{T}}_{o g}^{(-)} D_{g ; o}\right) P_{1}\left(\frac{2 \hat{\eta}}{\hat{\vartheta}_{2}}\right)^{2}\right. \\
& \left.+N_{o} \hat{T}_{o f}(-1)^{m_{2}} P_{2}\left(\frac{2 \hat{\eta}}{\hat{\vartheta_{2}}}\right)^{2}+N_{o} \hat{T}_{o h}(-1)^{m_{3}} P_{3}\left(\frac{2 \hat{\eta}}{\hat{\vartheta}_{2}}\right)^{2}\right\}
\end{aligned}
$$

The 99 sector gives a gauge group $\mathrm{SO}(16-a) \times \mathrm{SO}(a) \times \mathrm{SO}(16-c) \times \mathrm{SO}(c)$, with $\mathcal{N}=$ 1 supersymmetry and chiral multiplets in the representation $(16-a, a, 1,1)$ and in five additional ones differing by permutations of the entries. The $\overline{5}_{1} \overline{5}_{1}$ sector gives a gauge group $\operatorname{USp}(8) \times \operatorname{USp}(8)$, with complex scalars in the $(36,1)$ and $(1,36)$, Weyl spinors in two copies of the $(28,1)$ and $(1,28)$, and one $\mathcal{N}=2$ hypermultiplet in the $(8,8)$. Finally, the $9 \overline{5}_{1}$ sector gives Weyl spinors in the representations $(16-a, 1,1,1 ; 8,1),(1, a, 1,1 ; 8,1),(1,1,16-$ $c, 1 ; 1,8),(1,1,1, c ; 1,8)$ and complex scalars in the representations $(16-a, 1,1,1 ; 1,8)$, $(1, a, 1,1 ; 1,8),(1,1,16-c, 1 ; 8,1),(1,1,1, c ; 8,1)$. In the decompactification limit $R_{2}, R_{3} \rightarrow$ $\infty$, local tadpole cancellation requires a further breaking of the $\bar{D} \overline{5}$ gauge group to $\operatorname{USp}(4)^{4}$, and the resulting configuration may be linked to the $Z_{2}$ orientifold discussed in [10]. 


\section{Open descendants of the $T^{6} / Z_{4}$ orbifold}

The $Z_{4}$ orbifold is obtained identifying the complex world-sheet coordinates $X^{a}$ on the three internal tori and the world-sheet fermions $\lambda^{a}$ according to $X^{a} \sim \omega_{a} X^{a}, \lambda^{a} \sim \omega_{a} \lambda^{a}$, where $\omega_{a}=e^{2 \pi i t_{a}}(a=1,2,3)$ and the twist vector has components $\left(t_{1}, t_{2}, t_{3}\right)=\left(\frac{1}{4}, \frac{1}{4},-\frac{1}{2}\right)$. In order to display the contributions of the $\lambda^{a}$, it is convenient to decompose the original level-one $\mathrm{SO}(8)$ characters with respect to $\mathrm{SO}(2) \times \mathrm{SO}(2) \times \mathrm{SU}(2) \times \mathrm{U}(1)$. To this end, we introduce the two level-one $\mathrm{SU}(2)$ characters $\left(\chi_{0}, \chi_{1 / 2}\right)$, of conformal weights $\left(0, \frac{1}{4}\right)$, and the eight $\mathrm{U}(1)$ characters $\xi_{m}(m=0, \pm 1, \pm 2, \pm 3,4)$ for a boson on a circle of radius $R=\sqrt{8}$, of conformal weights $h_{m}=m^{2} / 16$. The contribution of the $\lambda^{a}$ to the sector twisted by $\omega^{k}$ and projected by $\omega^{l},(k, l=0,1,2,3)$, can then be expressed as

$$
\psi_{k l}=\rho_{k 0}+i^{l} \rho_{k 1}+(-1)^{l} \rho_{k 2}+(-i)^{l} \rho_{k 3}
$$

where the characters $\rho_{k l}$ are collected in Appendix B, while the contributions of the internal (lattice) bosons are

$$
\phi_{k l}=\left[-2 \sin \left(\frac{\pi l}{4}\right)\right]^{\delta_{k, 0}} \frac{\eta}{\theta\left[\begin{array}{c}
1 / 2+k / 4 \\
1 / 2+l / 4
\end{array}\right]} .
$$

The torus amplitude is then

$$
\begin{aligned}
\mathcal{T} & =\frac{1}{4}\left\{\left|\psi_{00}\right|^{2} \Lambda_{1} \Lambda_{2} \Lambda_{3}+\psi_{01} \bar{\psi}_{03} \phi_{01}^{2} \bar{\phi}_{03}^{2}\left|\frac{2 \eta}{\vartheta_{2}}\right|^{2}+\left|\psi_{02}\right|^{2}\left|\frac{2 \eta}{\vartheta_{2}}\right|^{4} \Lambda_{3}+\psi_{03} \bar{\psi}_{01} \phi_{03}^{2} \bar{\phi}_{01}^{2}\left|\frac{2 \eta}{\vartheta_{2}}\right|^{2}\right. \\
& +16\left(\left|\psi_{20}\right|^{2}\left|\frac{\eta}{\vartheta_{4}}\right|^{4} \Lambda_{3}+\left|\psi_{22}\right|^{2}\left|\frac{\eta}{\vartheta_{3}}\right|^{4} \Lambda_{3}\right)+4\left(\psi_{21} \bar{\psi}_{23} \phi_{21}^{2} \bar{\phi}_{23}^{2}\left|\frac{2 \eta}{\vartheta_{2}}\right|^{2}+\psi_{23} \bar{\psi}_{21} \phi_{23}^{2} \bar{\phi}_{21}^{2}\left|\frac{2 \eta}{\vartheta_{2}}\right|^{2}\right) \\
& +16\left(\psi_{10} \bar{\psi}_{30} \phi_{10}^{2} \bar{\phi}_{30}^{2}\left|\frac{\eta}{\vartheta_{4}}\right|^{2}+\psi_{11} \bar{\psi}_{33} \phi_{11}^{2} \bar{\phi}_{33}^{2}\left|\frac{\eta}{\vartheta_{3}}\right|^{2}+\psi_{12} \bar{\psi}_{32} \phi_{12}^{2} \bar{\phi}_{32}^{2}\left|\frac{\eta}{\vartheta_{4}}\right|^{2}\right. \\
& +\psi_{13} \bar{\psi}_{31} \phi_{13}^{2} \bar{\phi}_{31}^{2}\left|\frac{\eta}{\vartheta_{3}}\right|^{2}+\psi_{30} \bar{\psi}_{10} \phi_{30}^{2} \bar{\phi}_{10}^{2}\left|\frac{\eta}{\vartheta_{4}}\right|^{2}+\psi_{31} \bar{\psi}_{13} \phi_{31}^{2} \bar{\phi}_{13}^{2}\left|\frac{\eta}{\vartheta_{3}}\right|^{2} \\
& \left.\left.+\psi_{32} \bar{\psi}_{12} \phi_{32}^{2} \bar{\phi}_{12}^{2}\left|\frac{\eta}{\vartheta_{4}}\right|^{2}+\psi_{33} \bar{\psi}_{11} \phi_{33}^{2} \bar{\phi}_{11}^{2}\left|\frac{\eta}{\vartheta_{3}}\right|^{2}\right)\right\} .
\end{aligned}
$$

Before the $\Omega$ projection, the massless sector describes $\mathcal{N}=2$ supergravity coupled to 7 vector multiplets and 32 hypermultiplets, and can thus be associated with the singular limit of a Calabi-Yau manifold with Hodge numbers $\left(h_{11}, h_{21}\right)=(31,7)$. 
Let us now turn to the construction of the open descendants, starting from the Klein bottle amplitude

$$
\begin{aligned}
\mathcal{K} & =\frac{1}{8}\left\{\psi_{00}\left(P_{1} P_{2} P_{3}+W_{1} W_{2} P_{3}\right)+2 \psi_{02}\left(\frac{2 \eta}{\vartheta_{2}}\right)^{2} W_{3}\right. \\
& \left.+\epsilon\left[2 \times 16 \psi_{20}\left(\frac{\eta}{\vartheta_{4}}\right)^{2} P_{3}+2 \times 4 \psi_{22}\left(\frac{\eta}{\vartheta_{3}}\right)^{2} W_{3}\right]\right\},
\end{aligned}
$$

where, as in the previous cases, we have inserted a sign $\epsilon= \pm 1$ in front of the twisted contributions. The choice $\epsilon=1$ gives the usual $\Omega$ projection, while the choice $\epsilon=-1$ inverts the charge of the $\mathrm{O} 5$ plane and, as in the previous examples, requires the introduction of antibranes. In the transverse channel, this amplitude turns into

$$
\begin{aligned}
\tilde{\mathcal{K}} & =\frac{2^{5}}{8}\left\{\psi_{00}\left(v_{1} v_{2} v_{3} W_{1}^{e} W_{2}^{e} W_{3}^{e}+\frac{v_{3}}{v_{1} v_{2}} P_{1}^{e} P_{2}^{e} W_{3}^{e}\right)+2 \psi_{20}\left(\frac{\eta}{\vartheta_{4}}\right)^{2} \frac{P_{3}^{e}}{v_{3}}\right. \\
& \left.+2 \epsilon \psi_{02}\left(\frac{2 \eta}{\vartheta_{2}}\right)^{2} W_{3}^{e} v_{3}-2 \epsilon \psi_{22}\left(\frac{\eta}{\vartheta_{3}}\right)^{2} \frac{P_{3}^{e}}{v_{3}}\right\},
\end{aligned}
$$

and a closer look at the contributions at the origin of the internal lattices

$$
\begin{aligned}
\tilde{\mathcal{K}}_{0} & =\frac{2^{5}}{8}\left\{\left(\sqrt{v_{1} v_{2} v_{3}}+\epsilon \sqrt{\frac{v_{3}}{v_{1} v_{2}}}\right)^{2}\left(\rho_{00}+\rho_{02}\right)+\left(\sqrt{v_{1} v_{2} v_{3}}-\epsilon \sqrt{\frac{v_{3}}{v_{1} v_{2}}}\right)^{2}\left(\rho_{01}+\rho_{03}\right)\right. \\
& \left.+\frac{4}{v_{3}} \frac{1}{2}\left[(1-\epsilon)\left(\rho_{20}+\rho_{22}\right)+(1+\epsilon)\left(\rho_{21}+\rho_{23}\right)\right]\right\}
\end{aligned}
$$

reveals the presence of a term proportional to $1 / v_{3}$. For $\epsilon=1$, this gives rise to a massless tadpole that can not be canceled by the annulus and Möbius contributions. For $\epsilon=-1$, however, this term becomes massive, and one can complete the construction of the open descendants without any further difficulties. In this case, the projected massless closed spectrum is $\mathcal{N}=1$ supergravity coupled to 6 vector multiplets and 33 chiral multiplets. For the open sector, it is convenient to introduce a compact notation, as in the previous Section, defining

$$
\tilde{\psi}_{k l}^{(\varepsilon)}=\psi_{k l}^{\mathrm{NS}}-\varepsilon \psi_{k l}^{\mathrm{R}}
$$

and denoting by

$$
\psi_{k l}^{(-)}=\sigma_{k 0}+i^{l} \sigma_{k 1}+(-1)^{l} \sigma_{k 2}+(-i)^{l} \sigma_{k 3}
$$


the combinations of characters obtained from $\tilde{\psi}_{l k}^{(-)}$after an $S$ modular transformation. The explicit definition of the $\sigma_{k l}$ may be found in Appendix B.

The transverse-channel annulus amplitude is then

$$
\begin{aligned}
\tilde{\mathcal{A}} & =\frac{2^{-5}}{8}\left\{\left(N^{2} v_{1} v_{2} v_{3} W_{1} W_{2} W_{3}+D^{2} \frac{v_{3}}{v_{1} v_{2}} P_{1} P_{2} W_{3}\right) \psi_{00}+32\left(R^{2}+R_{D}^{2}\right) \psi_{30} \phi_{30}^{2}\left(\frac{\eta}{\vartheta_{4}}\right)\right. \\
& +16\left(S^{2}+S_{D}^{2}\right) \psi_{20}\left(\frac{\eta}{\vartheta_{4}}\right)^{2} v_{3} W_{3}+32\left(T^{2}+T_{D}^{2}\right) \psi_{10} \phi_{10}^{2}\left(\frac{\eta}{\vartheta_{4}}\right) \\
& +2 N D \tilde{\psi}_{02}^{(-)}\left(\frac{2 \eta}{\vartheta_{2}}\right)^{2} v_{3} W_{3}+32 R R_{D} \tilde{\psi}_{32}^{(-)} \phi_{32}^{2}\left(\frac{\eta}{\vartheta_{4}}\right) \\
& \left.-8 S S_{D} \tilde{\psi}_{22}^{(-)}\left(\frac{\eta}{\vartheta_{3}}\right)^{2} v_{3} W_{3}+32 T T_{D} \tilde{\psi}_{12}^{(-)} \phi_{12}^{2}\left(\frac{\eta}{\vartheta_{4}}\right)\right\}
\end{aligned}
$$

and, in particular, the untwisted contributions at the origin of the lattices are

$$
\begin{aligned}
\tilde{\mathcal{A}}_{0} & =\frac{2^{-5}}{8}\left\{\left(N \sqrt{v_{1} v_{2} v_{3}}+D \sqrt{\frac{v_{3}}{v_{1} v_{2}}}\right)^{2}\left(\rho_{00}+\rho_{02}\right)^{\mathrm{NS}}\right. \\
& +\left(N \sqrt{v_{1} v_{2} v_{3}}-D \sqrt{\frac{v_{3}}{v_{1} v_{2}}}\right)^{2}\left(\rho_{01}+\rho_{03}\right)^{\mathrm{NS}} \\
& -\left(N \sqrt{v_{1} v_{2} v_{3}}-D \sqrt{\frac{v_{3}}{v_{1} v_{2}}}\right)^{2}\left(\rho_{00}+\rho_{02}\right)^{\mathrm{R}} \\
& \left.-\left(N \sqrt{v_{1} v_{2} v_{3}}+D \sqrt{\frac{v_{3}}{v_{1} v_{2}}}\right)^{2}\left(\rho_{01}+\rho_{03}\right)^{\mathrm{R}}\right\} .
\end{aligned}
$$

It should be appreciated that the structure of the breaking terms is consistent with eq. (2.12). Thus, in the sector twisted by $\omega^{2}$ they are

$$
\left(S+4 S_{D}\right)^{2}+15 S^{2}=16 S^{2}+16 S_{D}^{2}+8 S S_{D}
$$

since the branes are present only in one fixed torus, while in the sector twisted by $\omega$ (or $\left.\omega^{3}\right)$ they are

$$
4\left(R+2 R_{D}\right)^{2}+12 R^{2}=16 R^{2}+16 R_{D}^{2}+16 R R_{D}
$$

since the branes fill the third torus, and are thus present in four of the $\omega$-fixed points. 
As usual, the transverse-channel amplitudes $\tilde{\mathcal{K}}$ and $\tilde{\mathcal{A}}$ determine

$$
\begin{aligned}
\tilde{\mathcal{M}} & =-\frac{1}{4}\left\{N v_{1} v_{2} v_{3} W_{1}^{e} W_{2}^{e} W_{3}^{e} \hat{\psi}_{00}-N v_{3} W_{3}^{e} \hat{\psi}_{02}\left(\frac{2 \hat{\eta}}{\hat{\vartheta}_{2}}\right)^{2}-D \frac{v_{3}}{v_{1} v_{2}} P_{1}^{e} P_{2}^{e} W_{3}^{e} \hat{\tilde{\psi}}_{00}^{(-)}\right. \\
& +D v_{3} W_{3}^{e} \hat{\tilde{\psi}}_{02}^{(-)}\left(\frac{2 \hat{\eta}}{\hat{\vartheta}_{2}}\right)^{2}+2\left(S \hat{\psi}_{21}-S_{D} \hat{\tilde{\psi}}_{21}^{(-)}\right) \hat{\phi}_{21}^{2}\left(\frac{2 \hat{\eta}}{\hat{\vartheta}_{2}}\right) \\
& \left.+2\left(S \hat{\psi}_{23}-S_{D} \tilde{\tilde{\psi}}_{23}^{(-)}\right) \hat{\phi}_{23}^{2}\left(\frac{2 \hat{\eta}}{\hat{\vartheta}_{2}}\right)\right\},
\end{aligned}
$$

and the tadpole conditions, now solvable, are

$$
N=D=32, \quad R=R_{D}=S=S_{D}=T=T_{D}=0 .
$$

After $S$ and $P$ transformations, one can then recover from (3.9) and (3.13) the directchannel amplitudes

$$
\begin{aligned}
\mathcal{A} & =\frac{1}{8}\left\{\left(N^{2} P_{1} P_{2} P_{3}+D^{2} W_{1} W_{2} P_{3}\right) \psi_{00}+\left(R^{2}+R_{D}^{2}\right) \psi_{01} \phi_{01}^{2}\left(\frac{2 \eta}{\vartheta_{2}}\right)\right. \\
& +\left(S^{2}+S_{D}^{2}\right) \psi_{02}\left(\frac{2 \eta}{\vartheta_{2}}\right)^{2} P_{3}+\left(T^{2}+T_{D}^{2}\right) \psi_{03} \phi_{03}^{2}\left(\frac{2 \eta}{\vartheta_{2}}\right)+2 N D \psi_{20}^{(-)}\left(\frac{\eta}{\vartheta_{4}}\right)^{2} P_{3} \\
& \left.+2 R R_{D} \psi_{21}^{(-)} \phi_{21}^{2}\left(\frac{2 \eta}{\vartheta_{2}}\right)+2 S S_{D} \psi_{22}^{(-)}\left(\frac{\eta}{\vartheta_{3}}\right)^{2} P_{3}+2 T T_{D} \psi_{23}^{(-)} \phi_{23}^{2}\left(\frac{2 \eta}{\vartheta_{2}}\right)\right\}
\end{aligned}
$$

and

$$
\begin{aligned}
\mathcal{M} & =-\frac{1}{8}\left\{N P_{1} P_{2} P_{3} \hat{\psi}_{00}+N P_{3} \hat{\psi}_{02}\left(\frac{2 \hat{\eta}}{\hat{\vartheta}_{2}}\right)^{2}-D W_{1} W_{2} P_{3} \hat{\widetilde{\psi}}_{00}^{(-)}-D P_{3} \tilde{\tilde{\psi}}_{02}^{(-)}\left(\frac{2 \hat{\eta}}{\hat{\vartheta}_{2}}\right)^{2}\right. \\
& \left.+S \hat{\psi}_{01} \hat{\phi}_{01}^{2}\left(\frac{2 \hat{\eta}}{\hat{\vartheta}_{2}}\right)+S \hat{\psi}_{03} \hat{\phi}_{03}^{2}\left(\frac{2 \hat{\eta}}{\hat{\vartheta}_{2}}\right)-S_{D} \hat{\tilde{\psi}}_{01}^{(-)} \hat{\phi}_{01}^{2}\left(\frac{2 \hat{\eta}}{\hat{\vartheta}_{2}}\right)-S_{D} \hat{\tilde{\psi}}_{03}^{(-)} \hat{\phi}_{03}^{2}\left(\frac{2 \hat{\eta}}{\hat{\vartheta}_{2}}\right)\right\} \cdot
\end{aligned}
$$

As usual, the coefficients in $\tilde{\mathcal{M}}$ are fixed by factorization in the tube channel. Some sign ambiguities apparently present for massive characters, whose coefficients are not constrained by tadpoles, are fixed demanding that the direct-channel amplitudes have a correct particle interpretation. The latter calls for the parametrization

$$
\begin{aligned}
N & =n+m+p+\bar{m}, & D & =d+r+q+\bar{r}, \\
R & =n+i m-p-i \bar{m}, & R_{D} & =d+i r-q-i \bar{r},
\end{aligned}
$$




$$
\begin{aligned}
S=n-m+p-\bar{m}, & S_{D}=d-r+q-\bar{r}, \\
T=n-i m-p+i \bar{m}, & T_{D}=d-i r-q+i \bar{r},
\end{aligned}
$$

that implements the $Z_{4}$ orbifold projections on the Chan-Paton charges.

One can now read the massless open spectrum from the amplitudes (3.15), (3.16) restricted to the origin of the internal lattice

$$
\begin{aligned}
\mathcal{A}_{0}+\mathcal{M}_{0} & =\frac{1}{2}\left\{\rho_{00}\left(n^{2}+p^{2}+2 m \bar{m}+d^{2}+q^{2}+2 r \bar{r}\right)+\rho_{01}(2 n \bar{m}+2 p m+2 d \bar{r}+2 q r)\right. \\
& +\rho_{02}\left(2 n p+m^{2}+\bar{m}^{2}+2 d q+r^{2}+\bar{r}^{2}\right)+\rho_{03}(2 n m+2 p \bar{m}+2 d r+2 q \bar{r}) \\
& +\sigma_{20}(n d+p q+m \bar{r}+\bar{m} r)+\sigma_{21}(n \bar{r}+m q+p r+\bar{m} d) \\
& \left.+\sigma_{22}(n q+m r+p d+\bar{m} \bar{r})+\sigma_{23}(n r+m d+p \bar{r}+\bar{m} q)\right\} \\
& -\frac{1}{2}\left\{\hat{\rho}_{00}(n+p)-\hat{\tilde{\rho}}_{00}^{(-)}(d+q)+\hat{\rho}_{02}(m+\bar{m})-\hat{\tilde{\rho}}_{02}^{(-)}(r+\bar{r})\right\}
\end{aligned}
$$

while the tadpole conditions select the gauge group $\mathrm{SO}(8)_{9} \times \mathrm{SO}(8)_{9} \times \mathrm{U}(8)_{9} \times \mathrm{USp}(8)_{5} \times$ $\mathrm{USp}(8)_{\overline{5}} \times \mathrm{U}(8)_{\overline{5}}$. The D9 spectrum has $\mathcal{N}=1$ supersymmetry, with two chiral multiplets in the $(8,1, \overline{8})$ and in the $(1,8,8)$ representations, and one chiral multiplet in each of the $(1,1,28),(1,1, \overline{28})$ and $(8,8,1)$ representations. On the other hand, the D $\overline{5}$ spectrum is not supersymmetric, and contains, aside from the corresponding gauge bosons, spinors in the $(28,1,1),(1,28,1),(1,1,64),(1,1,28),(1,1, \overline{28})$, complex scalars in the $(1,1,36),(1,1, \overline{36})$, and chiral multiplets in the $(8,8,1)$ and in two copies of the $(8,1, \overline{8})$ and $(1,8,8)$. Finally, the $N D$ strings contain complex scalars in the $(8,1,1 ; 8,1,1),(1,8,1 ; 1,8,1),(1,1,8 ; 1,1, \overline{8})$ and $(1,1, \overline{8} ; 1,1,8)$, and Weyl spinors in the $(8,1,1 ; 1,1, \overline{8}),(1,1,8 ; 1,8,1),(1,8,1 ; 1,1,8)$ and $(1,1, \overline{8} ; 8,1,1)$. This spectrum is chiral, but is free of irreducible gauge anomalies. As in previous examples, if suitable diagonal subgroups of the D $\overline{5}$ factors are regarded as global symmetries, the D9 spectrum has $\mathcal{N}=1$ supersymmetry. 


\section{Type I vacua with branes and antibranes of the same type}

In the previous examples we have seen how the structure of the closed sector determines both the types and the total numbers of D-branes present in the open descendants. In these cases, the breaking of supersymmetry on the branes is directly enforced by the consistency of the model.

Somewhat different scenarios have been recently proposed in [17, 18, 20]. In the resulting models, a supersymmetric open sector is deformed allowing for the simultaneous presence of branes and antibranes of the same type. Whereas tadpole conditions only fix the total RR charge, the option of saturating it by a single type of D-brane, whenever available, stands out as the only one compatible with space-time supersymmetry. However, if one relaxes this last condition, there are no evident obstructions to considering vacuum configurations where branes and antibranes with a fixed total RR charge are simultaneously present. The rules for constructing this wider class of models can be simply presented referring to a ten-dimensional example.

The starting point is the familiar supersymmetric type IIB torus amplitude

$$
\mathcal{T}=\left|V_{8}-S_{8}\right|^{2}
$$

and the corresponding Klein bottle projection

$$
\mathcal{K}=\frac{1}{2}\left(V_{8}-S_{8}\right)
$$

In the transverse channel, the latter becomes

$$
\tilde{\mathcal{K}}=\frac{2^{5}}{2}\left(V_{8}-S_{8}\right)
$$

and requires an open sector with a net number of 32 branes in order to cancel the resulting RR tadpole. Actually, both $V_{8}$ and $S_{8}$ develop tadpoles in this case, that in the usual

type I model are related by supersymmetry, but are conceptually quite different. While 
NS-NS tadpoles result in redefinitions of the vacuum configuration, RR tadpoles signal in general genuine inconsistencies, and their presence is a symptom of the emergence of serious pathologies [6].

Actually, one can conceive a more general construction in this case [17], allowing in the transverse-channel annulus different reflection coefficients for the $V_{8}$ and $S_{8}$ characters, so that

$$
\tilde{\mathcal{A}}=\frac{2^{-5}}{2}\left[\left(n_{+}+n_{-}\right)^{2} V_{8}-\left(n_{+}-n_{-}\right)^{2} S_{8}\right]
$$

where $n_{+}$and $n_{-}$actually count the total numbers of D9 and D $\overline{9}$ branes. It should be appreciated how their relative minus sign in the coefficient of $S_{8}$ accounts neatly for their opposite RR charges, while they have clearly identical couplings to the graviton, consistently with the coefficient of $V_{8}$. The direct-channel annulus amplitude

$$
\mathcal{A}=\frac{n_{+}^{2}+n_{-}^{2}}{2}\left(V_{8}-S_{8}\right)+n_{+} n_{-}\left(O_{8}-C_{8}\right)
$$

reflects the opposite GSO projections for open strings stretched between two D-branes of the same type (99 or $\overline{9} \overline{9})$ and of different types (9 $\overline{9}$ or $\overline{9}$ ) [21. While the former yields the supersymmetric type I spectrum, the latter eliminates the vector and its spinorial superpartners, while retaining the tachyon and the spinor of opposite chirality. As a result, supersymmetry is broken and an instability, signaled by the presence of the tachyonic ground state, emerges.

The Möbius amplitude

$$
\mathcal{M}= \pm \frac{1}{2}\left(n_{+}+n_{-}\right) \hat{V}_{8}+\frac{1}{2}\left(n_{+}-n_{-}\right) \hat{S}_{8}
$$

now involves naturally an undetermined sign for $V_{8}$, whose tadpole is generally incompatible with the one of $S_{8}$, and is to be relaxed. Together with $\mathcal{A}$, the two signs lead to symplectic or orthogonal gauge groups with $S$ fermions in (anti)symmetric representations and tachyons and $C$ fermions in bi-fundamentals. 
In these ten-dimensional models, the only way to eliminate the tachyon consists in introducing only D9-branes. Depending on the signs in the Möbius amplitude, one thus recovers either the $\mathrm{SO}(32)$ superstring or the $\operatorname{USp}(32)$ model of [17]. On the other hand, more can be done if one compactifies the theory on some internal manifold. In this case, one can introduce Wilson lines (or, equivalently, separate the branes) in such a way that in the open strings stretched between separate 9 and $\overline{9}$ branes the tachyon actually becomes massive. It is instructive to analyze in some detail the simple case of circle compactification. As in the previous example, the Klein bottle amplitude is not affected, and is given by

$$
\mathcal{K}=\frac{1}{2}\left(V_{8}-S_{8}\right) P
$$

where $P$ denotes the sum over momentum states. However, the Wilson line affects the annulus amplitude, that in the transverse-channel now reads

$$
\tilde{\mathcal{A}}=\frac{2^{-5}}{2}\left[\left(n_{+}+(-1)^{n} n_{-}\right)^{2} V_{8}-\left(n_{+}-(-1)^{n} n_{-}\right)^{2} S_{8}\right] W
$$

where $(-1)^{n} W$ denotes an oscillating winding sum. As a result, in the direct channel amplitude

$$
\mathcal{A}=\frac{n_{+}^{2}+n_{-}^{2}}{2}\left(V_{8}-S_{8}\right) P+n_{+} n_{-}\left(O_{8}-C_{8}\right) P^{(1 / 2)},
$$

where $P^{(1 / 2)}$ denotes a sum over $\frac{1}{2}$-shifted momentum states, both the tachyon and the $C$ spinor are lifted. The open sector is completed by the Möbius amplitude

$$
\mathcal{M}=\frac{1}{2}\left[ \pm\left(n_{+}+n_{-}\right) \hat{V}_{8}+\left(n_{+}-n_{-}\right) \hat{S}_{8}\right] P
$$

and at the massless level comprises gauge bosons in the adjoint of $\mathrm{SO}\left(n_{+}\right) \times \mathrm{SO}\left(n_{-}\right)$(or $\operatorname{USp}\left(n_{+}\right) \times \operatorname{USp}\left(n_{-}\right)$, depending on the sign of $\hat{V}_{8}$ in $\left.\mathcal{M}\right)$ and $S$ spinors in (anti)symmetric representations.

\subsection{Toroidal compactifications with nine and five (anti)branes}

Let us now turn to six-dimensional toroidal compactifications. In this case, one has the interesting option to introduce in the standard type I model of 26] pairs of D5-D $\overline{5}$ and 
D9-D $\overline{9}$ branes. The result includes a chiral spectrum confined to the (non-supersymmetric) branes, and calls for the introduction of six-dimensional Green-Schwarz couplings to the single tensor present in the projected closed spectrum to cancel the residual gauge and mixed anomalies.

The starting point in this construction is the standard type IIB string compactified on a four-torus, for which

$$
\mathcal{T}=\left|V_{8}-S_{8}\right|^{2} \Lambda^{4}=\left|V_{4} O_{4}+O_{4} V_{4}-C_{4} C_{4}-S_{4} S_{4}\right|^{2} \Lambda^{4}
$$

One can now add the standard Klein bottle projection

$$
\mathcal{K}=\frac{1}{2}\left(V_{8}-S_{8}\right) P^{4}=\frac{1}{2}\left(V_{4} O_{4}+O_{4} V_{4}-C_{4} C_{4}-S_{4} S_{4}\right) P^{4}
$$

where, for later convenience, we have explicitly decomposed the $\mathrm{SO}(8)$ characters into products of $\mathrm{SO}(4)$ ones. The transverse-channel annulus amplitude

$$
\begin{aligned}
\tilde{\mathcal{A}} & =\frac{2^{-5}}{2}\left\{\left(V_{4} O_{4}+O_{4} V_{4}-C_{4} C_{4}-S_{4} S_{4}\right)\left[\left(N_{+}^{2}+N_{-}^{2}\right) v W^{4}+\left(D_{+}^{2}+D_{-}^{2}\right) \frac{1}{v} P^{4}\right]\right. \\
& +2\left(V_{4} O_{4}+O_{4} V_{4}+C_{4} C_{4}+S_{4} S_{4}\right)\left[N_{+} N_{-} v W^{3}(-)^{n} W+D_{+} D_{-} \frac{1}{v} P^{3}(-)^{m} P\right] \\
& +2\left(V_{4} O_{4}-O_{4} V_{4}-C_{4} C_{4}+S_{4} S_{4}\right)\left(\frac{2 \eta}{\vartheta_{2}}\right)^{2}\left(N_{+} D_{+}+N_{-} D_{-}\right) \\
& \left.+2\left(V_{4} O_{4}-O_{4} V_{4}+C_{4} C_{4}-S_{4} S_{4}\right)\left(\frac{2 \eta}{\vartheta_{2}}\right)^{2}\left(N_{+} D_{-}+N_{-} D_{+}\right)\right\}
\end{aligned}
$$

is somewhat unconventional, and thus deserves some comments. Actually, the 99, $\overline{9} \overline{9}, 9 \overline{9}$, $55, \overline{5} \overline{5}$ and $5 \overline{5}$ contributions have already been discussed previously and do not need further explanations, but one should notice the presence of Wilson lines (brane displacements) in the $9 \overline{9}(5 \overline{5})$ sectors. These are to affect different directions, and are needed to lift the tachyons that would otherwise be present in the open spectrum. The transverse-channel amplitude $\tilde{\mathcal{A}}$, however, includes additional mixed terms, absent in ordinary toroidal constructions, that are to be interpreted as orbifold-like projections. In fact, the simultaneous 
presence of 9 and 5-branes, a familiar feature of orbifold models, halves the number of supersymmetries and results in the corresponding $\left(Z_{2}\right)$ breaking of the characters $V_{8}$ and $S_{8}$. Of course, due to the simultaneous presence of branes and antibranes, the complete theory is not supersymmetric, as implied by the different signs in the RR sectors. The orbifold-like structure of the annulus amplitude can be better appreciated after an $S$ transformation to the direct-channel amplitude

$$
\begin{aligned}
\mathcal{A} & =\frac{1}{2}\left(V_{4} O_{4}+O_{4} V_{4}-C_{4} C_{4}-S_{4} S_{4}\right)\left[\left(N_{+}^{2}+N_{-}^{2}\right) P^{4}+\left(D_{+}^{2}+D_{-}^{2}\right) W^{4}\right] \\
& +\left(O_{4} O_{4}+V_{4} V_{4}-S_{4} C_{4}-C_{4} S_{4}\right)\left[N_{+} N_{-} P^{3} P^{(1 / 2)}+D_{+} D_{-} W^{3} W^{(1 / 2)}\right] \\
& +\left(O_{4} C_{4}-S_{4} O_{4}+V_{4} S_{4}-C_{4} V_{4}\right)\left(\frac{\eta}{\vartheta_{4}}\right)^{2}\left(N_{+} D_{+}+N_{-} D_{-}\right) \\
& +\left(O_{4} S_{4}-C_{4} O_{4}+V_{4} C_{4}-S_{4} V_{4}\right)\left(\frac{\eta}{\vartheta_{4}}\right)^{2}\left(N_{+} D_{-}+N_{-} D_{+}\right)
\end{aligned}
$$

While the mixed $N D$ terms have the structure familiar from $Z_{2}$ orbifolds, no breaking terms are actually present in this case both for $N$ and $D$ charges. This is precisely as demanded by the consistency conditions for open-string constructions, since only the toroidal bulk states are allowed to propagate in the transverse channel.

One can now derive the transverse-channel Möbius amplitude combining the terms in $\tilde{\mathcal{K}}$ and $\tilde{\mathcal{A}}$ at the origin of the lattices, so that

$$
\begin{aligned}
\tilde{\mathcal{M}}_{0} & =\frac{2}{2} \sqrt{v}\left\{\epsilon \hat{V}_{4} \hat{O}_{4}\left[\left(N_{+}+N_{-}\right) \sqrt{v}+\left(D_{+}+D_{-}\right) \frac{1}{\sqrt{v}}\right]\right. \\
& +\epsilon \hat{O}_{4} \hat{V}_{4}\left[\left(N_{+}+N_{-}\right) \sqrt{v}-\left(D_{+}+D_{-}\right) \frac{1}{\sqrt{v}}\right] \\
& +\hat{C}_{4} \hat{C}_{4}\left[\left(N_{+}-N_{-}\right) \sqrt{v}+\left(D_{+}-D_{-}\right) \frac{1}{\sqrt{v}}\right] \\
& \left.+\hat{S}_{4} \hat{S}_{4}\left[\left(N_{+}-N_{-}\right) \sqrt{v}-\left(D_{+}-D_{-}\right) \frac{1}{\sqrt{v}}\right]\right\},
\end{aligned}
$$

and extract two NS-NS and two RR tadpole conditions for this class of models:

$$
V_{4} O_{4}: \quad\left(N_{+}+N_{-}+32 \epsilon\right) \sqrt{v}+\frac{\left(D_{+}+D_{-}\right)}{\sqrt{v}}=0
$$




$$
\begin{aligned}
O_{4} V_{4}: & \left(N_{+}+N_{-}+32 \epsilon\right) \sqrt{v}-\frac{\left(D_{+}+D_{-}\right)}{\sqrt{v}}=0 \\
C_{4} C_{4}: & \left(N_{+}-N_{-}-32\right) \sqrt{v}+\frac{\left(D_{+}-D_{-}\right)}{\sqrt{v}}=0 \\
S_{4} S_{4}: & \left(N_{+}-N_{-}-32\right) \sqrt{v}-\frac{\left(D_{+}-D_{-}\right)}{\sqrt{v}}=0
\end{aligned}
$$

The RR tadpole conditions demand that the net charge of the D9/D $\overline{9}$-branes, $N_{+}-N_{-}$, be equal to 32 , and that the numbers of D5 and D $\overline{5}$ branes be the same. It is then impossible to satisfy the NS-NS tadpoles, and as a result a potential is generated for the six-dimensional dilaton, $\phi_{6}$, and for the volume $v$ of the internal torus:

$$
V_{\mathrm{eff}} \sim e^{-\phi_{6}}\left[\left(N_{+}+N_{-}+32 \epsilon\right) \sqrt{v}+\frac{\left(D_{+}+D_{-}\right)}{\sqrt{v}}\right] .
$$

This potential has the peculiar property of stabilizing the internal volume to the value

$$
v_{0}=\frac{D_{+}+D_{-}}{N_{+}+N_{-}+32 \epsilon},
$$

while giving a mass to the corresponding (breathing-mode) field. This feature is common to all models with $9, \overline{9}, 5$ and $\overline{5}$ branes that we shall encounter in the following Sections.

The direct-channel Möbius amplitude

$$
\begin{aligned}
\mathcal{M} & =\frac{1}{2}\left\{\epsilon\left(N_{+}+N_{-}\right)\left(\hat{V}_{4} \hat{O}_{4}+\hat{O}_{4} \hat{V}_{4}\right) P^{4}-\epsilon\left(D_{+}+D_{-}\right)\left(\hat{V}_{4} \hat{O}_{4}-\hat{O}_{4} \hat{V}_{4}\right)\left(\frac{2 \hat{\eta}}{\hat{\vartheta}_{2}}\right)^{2}\right. \\
& \left.+\left(N_{+}-N_{-}\right)\left(\hat{C}_{4} \hat{C}_{4}+\hat{S}_{4} \hat{S}_{4}\right) P^{4}-\left(D_{+}-D_{-}\right)\left(\hat{C}_{4} \hat{C}_{4}-\hat{S}_{4} \hat{S}_{4}\right)\left(\frac{2 \hat{\eta}}{\hat{\vartheta}_{2}}\right)^{2}\right\}
\end{aligned}
$$

follows from (4.15) after one includes the massive contributions and performs a $P$ transformation. The massless open spectrum depends on the sign $\epsilon$, that is not fixed by tadpole conditions. For $\epsilon=-1$, it comprises gauge bosons in the adjoint of $\mathrm{SO}\left(N_{+}\right)_{9} \times \mathrm{SO}\left(N_{-}\right) \overline{9}$ $\times \operatorname{USp}\left(D_{+}\right)_{5} \times \operatorname{USp}\left(D_{-}\right)_{\overline{5}}$, with the matter

$$
\begin{aligned}
& 4 \text { scalars }:(A, 1 ; 1,1), \quad(1, A ; 1,1), \quad(1,1 ; A, 1), \quad(1,1 ; 1, A), \\
& 2 \text { scalars }:(f, 1 ; f, 1), \quad(1, f ; 1, f), \quad(1, f ; f, 1), \quad(f, 1 ; 1, f),
\end{aligned}
$$




$\begin{aligned} & \text { L-spinor }:(A, 1 ; 1,1), \quad(1, S ; 1,1), \quad(1,1 ; S, 1), \quad(1,1 ; 1, A), \\ & \text { R-spinor }:(A, 1 ; 1,1), \quad(1, S ; 1,1), \quad(1,1 ; A, 1), \quad(1,1 ; 1, S), \\ & \text { half L-spinor } \quad(1, f ; f, 1), \quad(f, 1 ; 1, f), \\ & \text { half R-spinor }:(f, 1 ; f, 1), \quad(1, f ; 1, f),\end{aligned}$

where $S(A)$ and $f$ denote the (anti)symmetric and fundamental representations. On the other hand, for $\epsilon=+1$ symmetric and antisymmetric representations are interchanged in the bosonic sector (thus also interchanging orthogonal and symplectic gauge groups), while the massless closed states, still supersymmetric, fill the $\mathcal{N}=(1,1)$ supergravity multiplet. It should be appreciated that, to lowest order, after a toroidal compactification to four dimensions, for $\epsilon=+1(\epsilon=-1)$ the spectrum on the D9 and D5 (D $\overline{9}$ and D $\overline{5}$ ) branes has $\mathcal{N}=2$ supersymmetry, if suitable gauge subgroups for the D $\overline{9}$ and D $\overline{5}$ (D9 and D5) branes are regarded as global symmetries. Thus, to lowest order the resulting massless spectrum has $\mathcal{N}=4$ supersymmetry in the bulk, $\mathcal{N}=2$ supersymmetry on the branes (antibranes), while it is not supersymmetric on the antibranes (branes). The open sector is chiral and leads to residual mixed and gauge anomalies, summarized by the polynomial

$$
\mathcal{I}_{8}=\frac{1}{8}\left(\operatorname{tr} R^{2}-\operatorname{tr} F_{N_{+}}^{2}+\operatorname{tr} F_{N_{-}}^{2}\right)\left(\operatorname{tr} F_{D_{+}}^{2}-\operatorname{tr} F_{D_{-}}^{2}\right)
$$

and removed by Green-Schwarz couplings [27 involving the single antisymmetric tensor present in the massless closed spectrum, while the RR tadpole conditions guarantee the cancellation of all irreducible $\operatorname{tr} R^{4}$ and $\operatorname{tr} F^{4}$ anomalies.

One can further deform these spectra, allowing for a non-trivial quantized NS-NS $B_{i j}$ of rank $r$. Due to the peculiar structure of the models, the closed sector behaves as in standard toroidal compactifications [26], whereas the annulus amplitude, modified as in [28], in the transverse channel reads

$$
\tilde{\mathcal{A}}=\frac{2^{-5}}{2}\left\{( V _ { 4 } O _ { 4 } + O _ { 4 } V _ { 4 } - C _ { 4 } C _ { 4 } - S _ { 4 } S _ { 4 } ) \left[2^{r-4}\left(N_{+}^{2}+N_{-}^{2}\right) v \sum_{\left\{\varphi_{j}\right\}= \pm 1} \sum_{\left\{n_{i}\right\}} W_{n_{i}} e^{i \pi n_{i} B_{i j} \varphi_{j}}\right.\right.
$$




$$
\begin{aligned}
& \left.+\left(D_{+}^{2}+D_{-}^{2}\right) \frac{1}{v} \sum_{\left\{m_{i}\right\}} P_{m_{i}}\right] \\
& +2\left(V_{4} O_{4}+O_{4} V_{4}+C_{4} C_{4}+S_{4} S_{4}\right)\left[2^{r-4} N_{+} N_{-} v \sum_{\left\{\varphi_{j}\right\}= \pm 1} \sum_{\left\{n_{i}\right\}}(-1)^{n_{4}} W_{n_{i}} e^{i \pi n_{i} B_{i j} \varphi_{j}}\right. \\
& \left.+D_{+} D_{-} \frac{1}{v} \sum_{\left\{m_{i}\right\}}(-1)^{m_{3}} P_{m_{i}}\right] \\
& +2 \times 2^{r / 2}\left(V_{4} O_{4}-O_{4} V_{4}-C_{4} C_{4}+S_{4} S_{4}\right)\left(\frac{2 \eta}{\vartheta_{2}}\right)^{2}\left(N_{+} D_{+}+N_{-} D_{-}\right) \\
& \left.+2 \times 2^{r / 2}\left(V_{4} O_{4}-O_{4} V_{4}+C_{4} C_{4}-S_{4} S_{4}\right)\left(\frac{2 \eta}{\vartheta_{2}}\right)^{2}\left(N_{+} D_{-}+N_{-} D_{+}\right)\right\}
\end{aligned}
$$

The corresponding transverse-channel Möbius amplitude

$$
\begin{aligned}
\tilde{\mathcal{M}}= & \frac{2}{2}\left\{2^{(r-4) / 2} \epsilon\left(N_{+}+N_{-}\right)\left(V_{4} O_{4}+O_{4} V_{4}\right) v \sum_{\left\{\varphi_{j}\right\}= \pm 1} \sum_{\left\{n_{i}\right\}} W_{n_{i}} e^{i \pi n_{i} B_{i j} \varphi_{j}} \gamma_{\varphi}\right. \\
& +\epsilon\left(D_{+}+D_{-}\right)\left(V_{4} O_{4}-O_{4} V_{4}\right)\left(\frac{2 \eta}{\vartheta_{2}}\right)^{2} \\
& +2^{(r-4) / 2}\left(N_{+}-N_{-}\right)\left(C_{4} C_{4}+S_{4} S_{4}\right) v \sum_{\left\{\varphi_{j}\right\}= \pm 1} \sum_{\left\{n_{i}\right\}} W_{n_{i}} e^{i \pi n_{i} B_{i j} \varphi_{j}} \gamma_{\varphi} \\
& \left.+\epsilon\left(D_{+}-D_{-}\right)\left(C_{4} C_{4}-S_{4} S_{4}\right)\left(\frac{2 \eta}{\vartheta_{2}}\right)^{2}\right\}
\end{aligned}
$$

is thus fixed by factorization and, as usual, involves the signs $\gamma_{\varphi}$ that enforce a proper normalization. As a result, the net $\mathrm{RR}$ charge of the $\mathrm{D} 9 / \mathrm{D} \overline{9}$-branes is reduced by a factor $2^{r / 2}$ and, after a modular transformation to the direct channel, $2^{r / 2}$ copies of the $N D$ sectors are present, while the $\gamma_{\varphi}$ allow for continuous interpolations between orthogonal and symplectic gauge groups only on the D9 and D $\overline{9}$ branes.

\subsection{The $T^{4} / Z_{2}$ orbifold revisited}

Let us now reconsider the open descendants of the $T^{4} / Z_{2}$ orbifold, allowing for the simultaneous presence of branes and antibranes of the same type. For the sake of clarity, 
let us begin from the torus amplitude

$$
\mathcal{T}=\frac{1}{4}\left\{\left|Q_{o}+Q_{v}\right|^{2} \Lambda^{4}+\left|Q_{o}-Q_{v}\right|^{2}\left|\frac{2 \eta}{\vartheta_{2}}\right|^{4}+16\left|Q_{s}+Q_{c}\right|^{2}\left|\frac{\eta}{\vartheta_{4}}\right|^{4}+16\left|Q_{s}-Q_{c}\right|^{2}\left|\frac{\eta}{\vartheta_{3}}\right|^{4}\right\},
$$

and its Klein bottle projection

$$
\mathcal{K}=\frac{1}{4}\left\{\left(Q_{o}+Q_{v}\right)\left(P^{4}+W^{4}\right)+2 \times 16 \epsilon\left(Q_{s}+Q_{c}\right)\left(\frac{\eta}{\vartheta_{4}}\right)^{2}\right\},
$$

where we have used the standard $Z_{2}$ decompositions

$$
\begin{array}{ll}
Q_{o}=V_{4} O_{4}-C_{4} C_{4}, & Q_{v}=O_{4} V_{4}-S_{4} S_{4}, \\
Q_{s}=O_{4} C_{4}-S_{4} O_{4}, & Q_{c}=V_{4} S_{4}-C_{4} V_{4} .
\end{array}
$$

We have written $\mathcal{K}$ in this form, since one can actually modify the Klein bottle projection for twisted closed states, as in [10]. Multiple Klein-bottle projections, first discussed in [23], have also interesting physical applications to tachyon-free non-supersymmetric openstring models [29]. The choice $\epsilon=+1$ corresponds to the usual $T^{4} / Z_{2}$ orientifold, and gives a closed unoriented spectrum containing $\mathcal{N}=(1,0)$ supergravity coupled to 20 hypermultiplets and 1 tensor multiplet, while the choice $\epsilon=-1$ gives $\mathcal{N}=(1,0)$ supergravity coupled to 4 hypermultiplets and 17 tensor multiplets.

From the Klein-bottle contribution to the massless tadpoles

$$
\tilde{\mathcal{K}}_{0}=\frac{2^{5}}{4}\left\{\left(\sqrt{v}+\frac{\epsilon}{\sqrt{v}}\right)^{2} Q_{o}+\left(\sqrt{v}-\frac{\epsilon}{\sqrt{v}}\right)^{2} Q_{v}\right\}
$$

one can anticipate the need for a net number of 32 D9 and 32 (anti)D5-branes (for $\epsilon= \pm 1$, respectively) in order to cancel the $R R$ tadpoles.

Proceeding as in the previous Sections, the annulus amplitude in the presence of branes and antibranes is

$$
\mathcal{A}=\frac{1}{4}\left\{\left(V_{4} O_{4}+O_{4} V_{4}-C_{4} C_{4}-S_{4} S_{4}\right)\left[\left(N_{+}^{2}+N_{-}^{2}\right) P^{4}+\left(D_{+}^{2}+D_{-}^{2}\right) W^{4}\right]\right.
$$




$$
\begin{aligned}
& +2\left(O_{4} O_{4}+V_{4} V_{4}-S_{4} C_{4}-C_{4} S_{4}\right)\left[N_{+} N_{-} P^{3} P_{1 / 2}+D_{+} D_{-} W^{3} W_{1 / 2}\right] \\
& +\quad\left(V_{4} O_{4}-O_{4} V_{4}-C_{4} C_{4}+S_{4} S_{4}\right)\left(\frac{2 \eta}{\vartheta_{2}}\right)^{2}\left(R_{N_{+}}^{2}+R_{N_{-}}^{2}+R_{D_{+}}^{2}+R_{D_{-}}^{2}\right) \\
& +2\left(O_{4} C_{4}+V_{4} S_{4}-S_{4} O_{4}-C_{4} V_{4}\right)\left(\frac{\eta}{\vartheta_{4}}\right)^{2}\left(N_{+} D_{+}+N_{-} D_{-}\right) \\
& +2\left(O_{4} S_{4}+V_{4} C_{4}-C_{4} O_{4}-S_{4} V_{4}\right)\left(\frac{\eta}{\vartheta_{4}}\right)^{2}\left(N_{+} D_{-}+N_{-} D_{+}\right) \\
& +2\left(O_{4} C_{4}-V_{4} S_{4}-S_{4} O_{4}+C_{4} V_{4}\right)\left(\frac{\eta}{\vartheta_{3}}\right)^{2}\left(R_{N_{+}} R_{D_{+}}+R_{N_{-}} R_{D_{-}}\right) \\
& \left.+2\left(-O_{4} S_{4}+V_{4} C_{4}-C_{4} O_{4}+S_{4} V_{4}\right)\left(\frac{\eta}{\vartheta_{3}}\right)^{2}\left(R_{N_{+}} R_{D_{-}}+R_{N_{-}} R_{D_{+}}\right)\right\}
\end{aligned}
$$

where $N_{ \pm}\left(D_{ \pm}\right)$refer to the D9 and D $\overline{9}$ (D5 and D $\left.\overline{5}\right)$ branes, respectively, and the $R$ 's are orbifold-induced breaking terms. As in the previous cases, in order to lift the tachyons, following [18] we have inserted the D5 and D $\overline{5}$-branes at different fixed points of the orbifolds, and we have added Wilson lines for the D9-branes along a different coordinate.

Finally, the Möbius amplitude

$$
\begin{aligned}
\mathcal{M} & =-\frac{1}{4}\left\{\left(\hat{V}_{4} \hat{O}_{4}+\hat{O}_{4} \hat{V}_{4}-\hat{C}_{4} \hat{C}_{4}-\hat{S}_{4} \hat{S}_{4}\right)\left[N_{+} P^{4}+\epsilon D_{+} W^{4}\right]\right. \\
& +\left(\hat{V}_{4} \hat{O}_{4}+\hat{O}_{4} \hat{V}_{4}+\hat{C}_{4} \hat{C}_{4}+\hat{S}_{4} \hat{S}_{4}\right)\left[N_{-} P^{4}+\epsilon D_{-} W^{4}\right] \\
& -\left(\hat{V}_{4} \hat{O}_{4}-\hat{O}_{4} \hat{V}_{4}-\hat{C}_{4} \hat{C}_{4}+\hat{S}_{4} \hat{S}_{4}\right)\left(\frac{2 \hat{\eta}}{\hat{\vartheta}_{2}}\right)^{2}\left(\epsilon N_{+}+D_{+}\right) \\
& \left.-\left(\hat{V}_{4} \hat{O}_{4}-\hat{O}_{4} \hat{V}_{4}+\hat{C}_{4} \hat{C}_{4}-\hat{S}_{4} \hat{S}_{4}\right)\left(\frac{2 \hat{\eta}}{\hat{\vartheta}_{2}}\right)^{2}\left(\epsilon N_{-}+D_{-}\right)\right\}
\end{aligned}
$$

gives a proper symmetrization of the annulus amplitude, while the tadpole conditions

$$
\begin{aligned}
& N_{+}-N_{-}=32, \quad D_{+}-D_{-}=32 \epsilon \\
& R_{N_{+}}=R_{N_{-}}=R_{D_{+}}=R_{D_{-}}=0
\end{aligned}
$$

require net numbers of 32 D9-branes and 32 D5-branes (antibranes) for $\epsilon= \pm 1$.

The choice $\epsilon=+1$ corresponds to a deformation of the supersymmetric $\mathrm{U}(16) \times \mathrm{U}(16)$ 
model [30, 31], and requires the introduction of complex charges, so that

$$
\begin{array}{ll}
N_{ \pm}=n_{ \pm}+\bar{n}_{ \pm}, & R_{N_{ \pm}}=i\left(n_{ \pm}-\bar{n}_{ \pm}\right) \\
D_{ \pm}=d_{ \pm}+\bar{d}_{ \pm}, & R_{D_{ \pm}}=i\left(d_{ \pm}-\bar{d}_{ \pm}\right) .
\end{array}
$$

The resulting massless open spectrum can be extracted from

$$
\begin{aligned}
\mathcal{A}_{0} & +\mathcal{M}_{0}=\left(n_{+} \bar{n}_{+}+n_{-} \bar{n}_{-}+d_{+} \bar{d}_{+}+d_{-} \bar{d}_{-}\right)\left(V_{4} O_{4}-C_{4} C_{4}\right) \\
& +\frac{1}{2}\left(n_{+}^{2}+\bar{n}_{+}^{2}+n_{-}^{2}+\bar{n}_{-}^{2}+d_{+}^{2}+\bar{d}_{+}^{2}+d_{-}^{2}+\bar{d}_{-}^{2}\right)\left(O_{4} V_{4}-S_{4} S_{4}\right) \\
& +\left(n_{+} \bar{d}_{+}+\bar{n}_{+} d_{+}+n_{-} \bar{d}_{-}+\bar{n}_{-} d_{-}\right)\left(O_{4} C_{4}-S_{4} O_{4}\right) \\
& -\left(n_{+} \bar{d}_{-}+n_{-} \bar{d}_{+}+\bar{n}_{+} d_{-}+\bar{n}_{-} d_{+}\right) C_{4} O_{4}++\left(n_{+} d_{-}+\bar{n}_{+} \bar{d}_{-}+n_{-} d_{+}+\bar{n}_{-} \bar{d}_{+}\right) O_{4} S_{4} \\
& -\frac{1}{2}\left\{\left(n_{+}+\bar{n}_{+}+d_{+}+\bar{d}_{+}\right)\left(\hat{O}_{4} \hat{V}_{4}-\hat{S}_{4} \hat{S}_{4}\right)+\left(n_{-}+\bar{n}_{-}+d_{-}+\bar{d}_{-}\right)\left(\hat{O}_{4} \hat{V}_{4}+\hat{S}_{4} \hat{S}_{4}\right)\right\}
\end{aligned}
$$

The 99 and 55 sectors are supersymmetric, and comprise vector multiplets in the adjoint of $\mathrm{U}\left(n_{+}\right)_{9} \times \mathrm{U}\left(n_{-}\right)_{\overline{9}} \times \mathrm{U}\left(d_{+}\right)_{5} \times \mathrm{U}\left(d_{-}\right)_{\overline{5}}$, together with hypermultiplets in the antisymmetric and conjugate antisymmetric representations. On the other hand, the $\overline{9} \overline{9}$ and $\overline{5} \overline{5}$ are not supersymmetric and contain left-handed Weyl fermions in the antisymmetric and conjugate antisymmetric representations, and a quartet of real scalars in the symmetric and conjugate symmetric representations. Finally, the 95 and $\overline{9} \overline{5}$ sectors give hypermultiplets in the $\left(n_{+}, \bar{d}_{+}\right)$and $\left(n_{-}, \bar{d}_{-}\right)$, respectively, while the $9 \overline{5}$ and $\overline{9} 5$ sectors, evidently not supersymmetric, give a quartet of scalars in the $\left(n_{+}, d_{-}\right),\left(n_{-}, d_{+}\right)$and right-handed Weyl fermions in the $\left(n_{+}, \bar{d}_{-}\right),\left(n_{-}, \bar{d}_{+}\right)$. The irreducible gravitational and gauge anomalies vanish as a result of tadpole conditions, whereas the residual anomaly polynomial

$$
\mathcal{I}_{8}=-\frac{1}{16}\left[\operatorname{tr} R^{2}-2\left(\operatorname{tr} F_{N_{+}}^{2}-\operatorname{tr} F_{N_{-}}^{2}\right)\right]\left[\operatorname{tr} R^{2}-2\left(\operatorname{tr} F_{D_{+}}^{2}-\operatorname{tr} F_{D_{-}}^{2}\right)\right]
$$

requires a conventional Green-Schwarz mechanism [27] involving the single antisymmetric tensor present in the massless closed spectrum. 
The choice $\epsilon=-1$ corresponds to a deformation of the original model in [10], and calls for the parametrization

$$
\begin{array}{ll}
N_{ \pm}=n_{ \pm}+m_{ \pm}, & R_{N_{ \pm}}=n_{ \pm}-m_{ \pm}, \\
D_{ \pm}=p_{ \pm}+q_{ \pm}, & R_{D_{ \pm}}=p_{ \pm}-q_{ \pm},
\end{array}
$$

in terms of real charges. The massless open spectrum can now be read from

$$
\begin{aligned}
\mathcal{A}_{0} & +\mathcal{M}_{0}=\frac{1}{2}\left(n_{+}^{2}+n_{-}^{2}+m_{+}^{2}+m_{-}^{2}+p_{+}^{2}+p_{-}^{2}+q_{+}^{2}+q_{-}^{2}\right)\left(V_{4} O_{4}-C_{4} C_{4}\right) \\
& +\left(m_{+} n_{+}+m_{-} n_{-}+p_{+} q_{+}+p_{-} q_{-}\right)\left(O_{4} V_{4}-S_{4} S_{4}\right) \\
& +\left(m_{+} q_{+}+n_{+} p_{+}+m_{-} q_{-}+n_{-} p_{-}\right)\left(O_{4} C_{4}-S_{4} O_{4}\right) \\
& +\left(m_{+} p_{-}+n_{+} q_{-}+m_{-} p_{+}+n_{-} q_{+}\right) O_{4} S_{4}-\left(m_{+} q_{-}+n_{+} p_{-}+m_{-} q_{+}+n_{-} p_{+}\right) C_{4} O_{4} \\
& -\frac{1}{2}\left\{\left(n_{+}+m_{+}-p_{+}-q_{+}\right)\left(\hat{V}_{4} \hat{O}_{4}-\hat{C}_{4} \hat{C}_{4}\right)+\left(n_{-}+m_{-}-p_{-}-q_{-}\right)\left(\hat{V}_{4} \hat{O}_{4}+\hat{C}_{4} \hat{C}_{4}\right)\right\}
\end{aligned}
$$

and the massless excitations thus comprise gauge bosons in the adjoint of $\mathrm{SO}\left(m_{+}\right)_{9} \times$ $\mathrm{SO}\left(n_{+}\right)_{9} \times \mathrm{SO}\left(m_{-}\right)_{\overline{9}} \times \mathrm{SO}\left(n_{-}\right)_{\overline{9}} \times \mathrm{USp}\left(p_{+}\right)_{5} \times \operatorname{USp}\left(q_{+}\right)_{5} \times \operatorname{USp}\left(p_{-}\right)_{\overline{5}} \times \mathrm{USp}\left(q_{-}\right)_{\overline{5}}$, lefthanded Weyl fermions in the adjoint of $\mathrm{SO}\left(m_{+}\right), \operatorname{SO}\left(n_{+}\right), \operatorname{USp}\left(p_{+}\right)$and $\operatorname{USp}\left(q_{+}\right)$, in the symmetric representations of $\mathrm{SO}\left(m_{-}\right)$and $\mathrm{SO}\left(n_{-}\right)$and in the antisymmetric representations of $\operatorname{USp}\left(p_{-}\right)$and $\operatorname{USp}\left(q_{-}\right)$. They also comprise a full hypermultiplet in the $\left(m_{+}, n_{+}\right)$, $\left(m_{-}, n_{-}\right),\left(p_{+}, q_{+}\right),\left(p_{-}, q_{-}\right)$, as well as half hypermultiplets in the $\left(m_{+}, q_{+}\right),\left(n_{+}, p_{+}\right)$, $\left(m_{-}, q_{-}\right)$and $\left(n_{-}, p_{-}\right)$. Finally, the $9 \overline{5}$ and $5 \overline{9}$ sectors comprise complex scalars in the $\left(m_{+}, p_{-}\right),\left(n_{+}, q_{-}\right),\left(m_{-}, p_{+}\right)$and $\left(n_{-}, q_{+}\right)$, and left-handed symplectic Majorana-Weyl spinors in the $\left(m_{+}, q_{-}\right),\left(n_{+}, p_{-}\right),\left(m_{-}, q_{+}\right)$and $\left(n_{-}, p_{+}\right)$.

The tadpole conditions eliminate all irreducible gauge and gravitational anomalies, whereas the residual reducible anomaly

$$
\begin{aligned}
\mathcal{I}_{8} & =\frac{1}{64}\left[2 \operatorname{tr} R^{2}-\left(\operatorname{tr} F_{m_{+}}^{2}+\operatorname{tr} F_{n_{+}}^{2}-\operatorname{tr} F_{m_{-}}^{2}-\operatorname{tr} F_{n_{-}}^{2}-\operatorname{tr} F_{p_{+}}^{2}-\operatorname{tr} F_{q_{+}}^{2}+\operatorname{tr} F_{p_{-}}^{2}+\operatorname{tr} F_{q_{-}}^{2}\right)\right]^{2} \\
& -\frac{1}{64}\left(\operatorname{tr} F_{m_{+}}^{2}+\operatorname{tr} F_{n_{+}}^{2}-\operatorname{tr} F_{m_{-}}^{2}-\operatorname{tr} F_{n_{-}}^{2}+\operatorname{tr} F_{p_{+}}^{2}+\operatorname{tr} F_{q_{+}}^{2}-\operatorname{tr} F_{p_{-}}^{2}-\operatorname{tr} F_{q_{-}}^{2}\right)^{2}
\end{aligned}
$$




$$
\begin{aligned}
& +\frac{1}{64}\left(\operatorname{tr} F_{m_{+}}^{2}-\operatorname{tr} F_{n_{+}}^{2}-\operatorname{tr} F_{m_{-}}^{2}+\operatorname{tr} F_{n_{-}}^{2}-\operatorname{tr} F_{p_{+}}^{2}+\operatorname{tr} F_{q_{+}}^{2}+\operatorname{tr} F_{p_{-}}^{2}-\operatorname{tr} F_{q_{-}}^{2}\right)^{2} \\
& +\frac{4}{64}\left(\operatorname{tr} F_{m_{+}}^{2}-\operatorname{tr} F_{n_{+}}^{2}+\operatorname{tr} F_{m_{-}}^{2}-\operatorname{tr} F_{n_{-}}^{2}\right)^{2} \\
& +\frac{4}{64}\left(\operatorname{tr} F_{p_{+}}^{2}-\operatorname{tr} F_{q_{+}}^{2}+\operatorname{tr} F_{p_{-}}^{2}-\operatorname{tr} F_{q_{-}}^{2}\right)^{2} \\
& +\frac{3}{64}\left(\operatorname{tr} F_{m_{+}}^{2}-\operatorname{tr} F_{n_{+}}^{2}-\operatorname{tr} F_{m_{-}}^{2}+\operatorname{tr} F_{n_{-}}^{2}+\operatorname{tr} F_{p_{+}}^{2}-\operatorname{tr} F_{q_{+}}^{2}-\operatorname{tr} F_{p_{-}}^{2}+\operatorname{tr} F_{q_{-}}^{2}\right)^{2}
\end{aligned}
$$

can be removed by a generalized Green-Schwarz mechanism [32].

In both models a potential is generated for the six-dimensional dilaton and the volume of the internal manifold

$$
V_{\mathrm{eff}} \sim e^{-\phi_{6}}\left[\left(N_{+}+N_{-}-32\right) \sqrt{v}+\frac{\left(D_{+}+D_{-}-32 \epsilon\right)}{\sqrt{v}}\right],
$$

as a result of uncancelled NS-NS tadpoles. As in the previous model, this stabilizes the internal volume at a local minimum, that in this case is

$$
v_{0}=\frac{D_{+}+D_{-}-32 \epsilon}{N_{+}+N_{-}-32},
$$

and gives mass to the corresponding (breathing-mode) field.

In the present models discrete deformations for the NS-NS $B$-field may be introduced as in [26, 28], and result in a reduced total $R R$ charge for nine and five (anti)branes and in multiplicities for the $N D$ sectors. Moreover, the corresponding signs in the Möbius amplitude allow continuous interpolations between orthogonal (symplectic) and unitary gauge groups.

\subsection{The non-supersymmetric $T^{6} / Z_{4}$ orbifold revisited}

As a last example, we would like to generalize the non-supersymmetric $T^{6} / Z_{4}$ orbifold of Section 3, allowing for the simultaneous presence of branes and antibranes. As in the previous examples, the torus and Klein bottle amplitudes are not affected, whereas the annulus and Möbius amplitudes are now given by

$$
\mathcal{A}=\frac{1}{8}\left\{\left[\left(N_{+}^{2}+N_{-}^{2}\right) P_{1} P_{2} P_{3}+\left(D_{+}^{2}+D_{-}^{2}\right) W_{1} W_{2} P_{3}\right] \psi_{00}\right.
$$




$$
\begin{aligned}
& +\left(R_{N_{+}}^{2}+R_{N_{-}}^{2}+R_{D_{+}}^{2}+R_{D_{-}}^{2}\right) \psi_{01} \phi_{01}^{2}\left(\frac{2 \eta}{\vartheta_{2}}\right)+\left(S_{N_{+}}^{2}+S_{N_{-}}^{2}+S_{D_{+}}^{2}+S_{D_{-}}^{2}\right) \psi_{02}\left(\frac{2 \eta}{\vartheta_{2}}\right)^{2} P_{3} \\
& +\left(T_{N_{+}}^{2}+T_{N_{-}}^{2}+T_{D_{+}}^{2}+T_{D_{-}}^{2}\right) \psi_{03} \phi_{03}^{2}\left(\frac{2 \eta}{\vartheta_{2}}\right)+2\left(N_{+} N_{-} P_{1} P_{2}^{1 / 2} P_{3}+D_{+} D_{-} W_{1} W_{2}^{1 / 2} P_{3}\right) \psi_{00}^{(-)} \\
& +2\left(N_{+} D_{+}+N_{-} D_{-}\right) \psi_{20}\left(\frac{\eta}{\vartheta_{4}}\right)^{2} P_{3}+2\left(R_{N_{+}} R_{D_{+}}+R_{N_{-}} R_{D_{-}}\right) \psi_{21} \phi_{21}^{2}\left(\frac{2 \eta}{\vartheta_{2}}\right) \\
& +2\left(S_{N_{+}} S_{D_{+}}+R_{N_{-}} R_{D_{-}}\right) \psi_{22}\left(\frac{\eta}{\vartheta_{3}}\right)^{2} P_{3}+2\left(T_{N_{+}} T_{D_{+}}+R_{N_{-}} R_{D_{-}}\right) \psi_{23} \phi_{23}^{2}\left(\frac{2 \eta}{\vartheta_{2}}\right) \\
& +2\left(N_{+} D_{-}+N_{-} D_{+}\right) \psi_{20}^{(-)}\left(\frac{\eta}{\vartheta_{4}}\right)^{2} P_{3}+2\left(R_{N_{+}} R_{D_{-}}+R_{N_{-}} R_{D_{+}}\right) \psi_{21}^{(-)} \phi_{21}^{2}\left(\frac{2 \eta}{\vartheta_{2}}\right) \\
& \left.+2\left(S_{N_{+}} S_{D_{-}}+R_{N_{-}} R_{D_{+}}\right) \psi_{22}^{(-)}\left(\frac{\eta}{\vartheta_{3}}\right)^{2} P_{3}+2\left(T_{N_{+}} T_{D_{-}}+R_{N_{-}} R_{D_{+}}\right) \psi_{23}^{(-)} \phi_{23}^{2}\left(\frac{2 \eta}{\vartheta_{2}}\right)\right\}
\end{aligned}
$$

and

$$
\begin{aligned}
\mathcal{M} & =-\frac{1}{8}\left\{\left(N_{+} P_{1} P_{2} P_{3}-D_{+} W_{1} W_{2} P_{3}\right) \hat{\psi}_{00}+\left(N_{+}-D_{+}\right) P_{3} \hat{\psi}_{02}\left(\frac{2 \hat{\eta}}{\hat{\vartheta}_{2}}\right)^{2}\right. \\
& +\left(N_{-} P_{1} P_{2} P_{3}-D_{-} W_{1} W_{2} P_{3}\right) \tilde{\tilde{\psi}}_{00}^{(-)}+\left(N_{-}-D_{-}\right) P_{3} \tilde{\tilde{\psi}}_{02}^{(-)}\left(\frac{2 \hat{\eta}}{\hat{\vartheta}_{2}}\right)^{2} \\
& +2\left(S_{N_{+}}-S_{D_{+}}\right) \hat{\psi}_{01} \hat{\phi}_{01}^{2}\left(\frac{2 \hat{\eta}}{\hat{\vartheta}_{2}}\right)+2\left(S_{N_{+}}-S_{D_{+}}\right) \hat{\psi}_{03} \hat{\phi}_{03}^{2}\left(\frac{2 \hat{\eta}}{\hat{\vartheta}_{2}}\right) \\
& \left.+2\left(S_{N_{-}}-S_{D_{-}}\right) \hat{\tilde{\psi}}_{01}^{(-)} \hat{\phi}_{01}^{2}\left(\frac{2 \hat{\eta}}{\hat{\vartheta}_{2}}\right)+2\left(S_{N_{-}}-S_{D_{-}}\right) \hat{\tilde{\psi}}_{03}^{(-)} \hat{\phi}_{03}^{2}\left(\frac{2 \hat{\eta}}{\hat{\vartheta}_{2}}\right)\right\}
\end{aligned}
$$

where the $\psi$ 's and $\phi$ 's have been introduced in Section $3, P^{1 / 2}$ and $W^{1 / 2}$ denote shifted momentum and winding sums, and we are parametrizing again the $9, \overline{9}, 5$ and $\overline{5}$-brane charges by $N_{ \pm}, D_{ \pm}$and by their orbifold-induced breakings $R, S$ and $T$. The $Z_{4}$ action suggests the parametrization

$$
\begin{array}{cl}
N_{ \pm}=n_{ \pm}+m_{ \pm}+p_{ \pm}+\bar{m}_{ \pm}, & D_{ \pm}=q_{ \pm}+r_{ \pm}+s_{ \pm}+\bar{r}_{ \pm}, \\
R_{N_{ \pm}}=n_{ \pm}+i m_{ \pm}-p_{ \pm}-i \bar{m}_{ \pm}, & R_{D_{ \pm}}=q_{ \pm}+i r_{ \pm}-s_{ \pm}-i \bar{r}_{ \pm}, \\
S_{N_{ \pm}}=n_{ \pm}-m_{ \pm}+p_{ \pm}-\bar{m}_{ \pm}, & S_{D_{ \pm}}=q_{ \pm}-r_{ \pm}+s_{ \pm}-\bar{r}_{ \pm}, \\
T_{N_{ \pm}}=n_{ \pm}-i m_{ \pm}-p_{ \pm}+i \bar{m}_{ \pm}, & T_{D_{ \pm}}=q_{ \pm}-i r_{ \pm}-s_{ \pm}+i \bar{r}_{ \pm}
\end{array}
$$

and, as expected from the simpler case discussed in Section 3, the RR tadpole conditions 
fix to 32 the net numbers of D9-branes and D $\overline{5}$-branes, and require the vanishing of all the $R, S$ and $T$ breaking coefficients. Even in this case the NS-NS tadpole conditions can not be satisfied, and as a result the potential

$$
V_{\text {eff }} \sim e^{-\phi_{4}} \sqrt{v_{3}}\left[\left(N_{+}+N_{-}-32\right) \sqrt{v_{1} v_{2}}+\frac{\left(D_{+}+D_{-}+32\right)}{\sqrt{v_{1} v_{2}}}\right]
$$

is generated for the four-dimensional dilaton and the volume of the internal manifold. As in the previous cases, it constrains the internal volumes, since it has a minimum for

$$
\left(v_{1} v_{2}\right)_{0}=\frac{D_{+}+D_{-}+32}{N_{+}+N_{-}-32} .
$$

From the tadpole conditions and the massless contributions to $\mathcal{A}$ and $\mathcal{M}$, one finds the Chan-Paton gauge group $\mathrm{SO}\left(n_{+}\right)_{9} \times \mathrm{SO}\left(p_{+}\right)_{9} \times \mathrm{U}\left(m_{+}\right)_{9} \times \mathrm{SO}\left(n_{-}\right) \overline{9} \times \mathrm{SO}\left(p_{-}\right) \overline{9} \times \mathrm{U}\left(m_{-}\right)_{\overline{9}}$ $\times \mathrm{USp}\left(q_{+}\right)_{5} \times \mathrm{USp}\left(s_{+}\right)_{5} \times \mathrm{U}\left(r_{+}\right)_{5} \times \mathrm{USp}\left(q_{-}\right)_{\overline{5}} \times \mathrm{USp}\left(s_{-}\right)_{\overline{5}} \times \mathrm{U}\left(r_{-}\right)_{\overline{5}}$. The 99 and 55 sectors are supersymmetric and, aside from $\mathcal{N}=1$ vector multiplets in the adjoint of the gauge group, comprise chiral multiplets in the representations

$$
\begin{aligned}
& 99: \quad 2\left(n_{+}, \bar{m}_{+}\right), \quad 2\left(p_{+}, m_{+}\right), \quad\left(n_{+}, p_{+}\right), \quad A_{m_{+}}, \quad \bar{A}_{m_{+}}, \\
& 55: \quad 2\left(q_{+}, \bar{r}_{+}\right), \quad 2\left(s_{+}, r_{+}\right), \quad\left(q_{+}, s_{+}\right), \quad S_{r_{+}}, \quad \bar{S}_{r_{+}} .
\end{aligned}
$$

The $\overline{9} \overline{9}$ and $\overline{5} \overline{5}$ sectors are non-supersymmetric and, aside from the gauge bosons in the adjoint of the corresponding gauge groups, comprise Dirac spinors in the representations

$$
\begin{aligned}
& \overline{9} \overline{9}: \quad S_{n_{-}}, \quad S_{p_{-}}, \quad \operatorname{Adj}_{m_{-}}, \quad S_{m_{-}}, \quad \bar{S}_{m_{-}}, \\
& \overline{5} \overline{5}: \quad A_{q_{-}}, \quad A_{s_{-}}, \quad \operatorname{Adj}_{r_{-}}, \quad A_{r_{-}}, \bar{A}_{r_{-}},
\end{aligned}
$$

scalars in the representations $S_{m_{-}}$and $\bar{S}_{m_{-}}\left(A_{r_{-}}\right.$and $\left.\bar{A}_{r_{-}}\right)$, pairs of chiral multiplets in the representations $\left(n_{-}, \bar{m}_{-}\right)$and $\left(p_{-}, m_{-}\right)\left(\left(q_{-}, \bar{r}_{-}\right)\right.$and $\left.\left(s_{-}, r_{-}\right)\right)$, and a single chiral multiplet in the representation $\left(n_{-}, p_{-}\right)\left(\left(q_{-}, s_{-}\right)\right)$for the $\overline{9} \overline{9}(\overline{5} \overline{5})$ sectors. The $9 \overline{5}$ spectrum contains

\footnotetext{
${ }^{2}$ For the sake of brevity, we denote by $S_{\alpha}\left(A_{\alpha}\right)$ the (anti)symmetric representations for the $\alpha$-th factor in the gauge group, by $\bar{S}_{\alpha}\left(\bar{A}_{\alpha}\right)$ their conjugates, and by Adj the adjoint of unitary factors.
} 
scalars in the representations $\left(n_{+}, q_{-}\right),\left(p_{+}, s_{-}\right),\left(m_{+}, \bar{r}_{-}\right)$and $\left(\bar{m}_{+}, r_{-}\right)$, and spinors in the representations $\left(n_{+}, \bar{r}_{-}\right),\left(p_{+}, r_{-}\right),\left(m_{+}, s_{-}\right)$and $\left(\bar{m}_{+}, q_{-}\right)$, while the 59 sector contains chiral multiplets in the representations $\left(n_{+}, \bar{r}_{+}\right),\left(p_{+}, r_{+}\right),\left(m_{+}, s_{+}\right)$and $\left(\bar{m}_{+}, q_{+}\right)$. Finally, the $5 \overline{9}$ and $\overline{5} \overline{9}$ sectors give similar contributions, with suitable relabelings of the Chan-Paton charges. This chiral spectrum is free of non-abelian gauge anomalies.

\section{Conclusions}

In this paper we have discussed type I models where the basic two-dimensional consistency conditions (RR tadpoles), although apparently unsolvable, can actually be solved provided supersymmetry is broken at the string scale on some (anti)branes, while some amount of supersymmetry is left, to lowest order, on other branes, and most notably in the bulk (gravitational) sector.

This new feature of our mechanism (called in [10] brane supersymmetry breaking), where the breaking is tied to the string scale, is the main difference compared to other previously known mechanisms in heterotic strings [11], in type I strings [12, 14, 15, 33, 13, 34] or in M-theory [35]. Brane supersymmetry breaking is typically accompanied by uncanceled NS-NS tadpoles for the dilaton at some moduli fields of the compact manifold.

Since our models contain non-BPS brane-antibrane systems, one might wonder whether they correspond to stable configurations. This is indeed the case, and actually the "minimal" D9-D $\overline{5}$ pairs of [10], aside from having no open-string tachyons, experience no net mutual forces, as can be seen from the vanishing of the corresponding annulus amplitude. Following [17, 18], these models can be further deformed by the inclusion of additional brane-antibrane pairs of the same type (D9-D $\left.\overline{9}, \mathrm{D} 5_{i}-\mathrm{D} \overline{5}_{i}\right)$. We would like to stress that this additional deformation is an interesting option, not required by any RR tadpole conditions. Rather, it destroys local tadpole cancellations, while the new brane-antibrane pairs do experience mutual forces, although the tachyons resulting from strings stretched between them 
may be lifted if the endpoints are suitably separated in the internal space. Interestingly, the NS-NS tadpoles resulting from the various mutual interactions between (anti)branes and orientifold planes determine a scalar potential that can actually stabilize some of the radii of the compact space, giving masses to the corresponding fields. Large (small) volumes, however, require unnaturally large numbers of D5-D $\overline{5}$ (D9-D $\overline{9})$ pairs, whose presence, therefore, asks for a dynamical reason. Since the whole approach is perturbative, the dilaton potential has, not surprisingly, a runaway behavior towards vanishing string coupling. We would like to stress that all these constructions, despite their attractive features, do not solve the problem of the (four-dimensional) cosmological constant.

Breaking supersymmetry at the string scale is a viable phenomenological alternative if our world is a non-supersymmetric (anti)brane and the string scale $M_{I}$ is in the $\mathrm{TeV}$ range, or if our world is on a brane that, to lowest order, is supersymmetric. In the latter case, supersymmetry breaking could be mediated by gauge interactions, and a realistic spectrum would ask for a string scale of the order of $100 \mathrm{TeV}$ if the non-supersymmetric (anti)brane gauge coupling were of order one, or at an intermediate value if the (anti)brane gauge coupling were suppressed by the volume of the internal space. Alternatively, if this coupling were very tiny, gravitation would mediate supersymmetry breaking with an intermediate string scale of the order of $10^{11} \mathrm{GeV}$ [5]. The (tree-level) supersymmetry present in the bulk sector can have far-reaching physical consequences. Indeed, quantum corrections to brane/antibrane couplings can also be interpreted in terms of effective brane couplings to bulk fields. If these respect the bulk symmetry, one could contemplate the fascinating possibility of living in a non-supersymmetric world where quantum corrections are governed by a supersymmetric bulk sector. In this case, the gauge hierarchy would be protected and the quantum corrections would be very similar to those of a supersymmetric brane theory. We will return to these interesting issues in the near future. 


\section{Acknowledgments}

C.A. and A.S. would like to thank the Physics Department of the Humboldt University, C.A. and E.D. would like thank the Physics Department of the University of Rome "Tor Vergata", and A.S. would like to thank the Centre de Physique Théorique of the École Polytechnique for the warm hospitality extended to them while this work was in progress. This research was supported in part by the EEC under TMR contract ERBFMRX-CT960090, and in part by the National Science Foundation, under Grant No. PHY94-07194. 


\section{A Characters for the $T^{6} / Z_{2} \times Z_{2}$ orbifolds}

In this Appendix we list the $Z_{2} \times Z_{2}$ characters needed for the models in Section 2 . They may be expressed as ordered products of the four $\mathrm{SO}(2)$ level-one characters, $O_{2}, V_{2}$, $S_{2}$ and $C_{2}$ :

$$
\begin{aligned}
& \tau_{\text {oo }}=V_{2} \mathrm{O}_{2} \mathrm{O}_{2} \mathrm{O}_{2}+\mathrm{O}_{2} V_{2} V_{2} V_{2}-S_{2} S_{2} S_{2} S_{2}-C_{2} C_{2} C_{2} C_{2} \text {, } \\
& \tau_{\text {og }}=\mathrm{O}_{2} V_{2} \mathrm{O}_{2} \mathrm{O}_{2}+V_{2} \mathrm{O}_{2} V_{2} V_{2}-C_{2} C_{2} S_{2} S_{2}-S_{2} S_{2} C_{2} C_{2}, \\
& \tau_{\text {oh }}=\mathrm{O}_{2} \mathrm{O}_{2} \mathrm{O}_{2} V_{2}+V_{2} V_{2} V_{2} O_{2}-C_{2} S_{2} S_{2} C_{2}-S_{2} C_{2} C_{2} S_{2} \text {, } \\
& \tau_{\text {of }}=\mathrm{O}_{2} \mathrm{O}_{2} V_{2} \mathrm{O}_{2}+V_{2} V_{2} \mathrm{O}_{2} V_{2}-C_{2} S_{2} C_{2} S_{2}-S_{2} C_{2} S_{2} C_{2}, \\
& \tau_{g o}=V_{2} O_{2} S_{2} C_{2}+O_{2} V_{2} C_{2} S_{2}-S_{2} S_{2} V_{2} O_{2}-C_{2} C_{2} O_{2} V_{2} \\
& \tau_{g g}=\mathrm{O}_{2} V_{2} S_{2} C_{2}+V_{2} O_{2} C_{2} S_{2}-S_{2} S_{2} O_{2} V_{2}-C_{2} C_{2} V_{2} O_{2} \\
& \tau_{g h}=\mathrm{O}_{2} \mathrm{O}_{2} \mathrm{~S}_{2} \mathrm{~S}_{2}+\mathrm{V}_{2} \mathrm{~V}_{2} \mathrm{C}_{2} \mathrm{C}_{2}-\mathrm{C}_{2} \mathrm{~S}_{2} V_{2} V_{2}-\mathrm{S}_{2} \mathrm{C}_{2} \mathrm{O}_{2} \mathrm{O}_{2} \\
& \tau_{g f}=\mathrm{O}_{2} \mathrm{O}_{2} \mathrm{C}_{2} \mathrm{C}_{2}+\mathrm{V}_{2} \mathrm{~V}_{2} \mathrm{~S}_{2} \mathrm{~S}_{2}-\mathrm{S}_{2} \mathrm{C}_{2} V_{2} V_{2}-\mathrm{C}_{2} \mathrm{~S}_{2} \mathrm{O}_{2} \mathrm{O}_{2}, \\
& \tau_{h o}=V_{2} S_{2} C_{2} O_{2}+O_{2} C_{2} S_{2} V_{2}-C_{2} O_{2} V_{2} C_{2}-S_{2} V_{2} O_{2} S_{2}, \\
& \tau_{h g}=\mathrm{O}_{2} \mathrm{C}_{2} \mathrm{C}_{2} \mathrm{O}_{2}+V_{2} S_{2} S_{2} V_{2}-C_{2} \mathrm{O}_{2} O_{2} S_{2}-S_{2} V_{2} V_{2} C_{2}, \\
& \tau_{h h}=\mathrm{O}_{2} S_{2} C_{2} V_{2}+V_{2} C_{2} S_{2} O_{2}-S_{2} O_{2} V_{2} S_{2}-C_{2} V_{2} O_{2} C_{2}, \\
& \tau_{h f}=O_{2} S_{2} S_{2} O_{2}+V_{2} C_{2} C_{2} V_{2}-C_{2} V_{2} V_{2} S_{2}-S_{2} O_{2} O_{2} C_{2}, \\
& \tau_{f o}=V_{2} S_{2} O_{2} C_{2}+O_{2} C_{2} V_{2} S_{2}-S_{2} V_{2} S_{2} O_{2}-C_{2} O_{2} C_{2} V_{2}, \\
& \tau_{f g}=\mathrm{O}_{2} \mathrm{C}_{2} \mathrm{O}_{2} \mathrm{C}_{2}+\mathrm{V}_{2} \mathrm{~S}_{2} V_{2} \mathrm{~S}_{2}-\mathrm{C}_{2} \mathrm{O}_{2} \mathrm{~S}_{2} \mathrm{O}_{2}-\mathrm{S}_{2} V_{2} \mathrm{C}_{2} V_{2}, \\
& \tau_{f h}=\mathrm{O}_{2} S_{2} \mathrm{O}_{2} \mathrm{~S}_{2}+V_{2} C_{2} V_{2} C_{2}-C_{2} V_{2} S_{2} V_{2}-S_{2} O_{2} C_{2} O_{2}, \\
& \tau_{f f}=\mathrm{O}_{2} S_{2} V_{2} C_{2}+V_{2} C_{2} O_{2} S_{2}-C_{2} V_{2} C_{2} O_{2}-S_{2} O_{2} S_{2} V_{2} .
\end{aligned}
$$


While these are sufficient to describe all supersymmetric $Z_{2} \times Z_{2}$ amplitudes, when brane supersymmetry breaking is present additional characters are needed to describe the open strings stretched between branes and antibranes. These new characters, that we denote by $\tau_{k l}^{(-)}$, are obtained from the others in eq. (A1) interchanging $O_{2}$ with $V_{2}$ and $S_{2}$ with $C_{2}$ in the last three factors, that correspond to the three internal tori:

$$
\begin{aligned}
& \tau_{\text {oo }}^{(-)}=\mathrm{O}_{2} \mathrm{O}_{2} \mathrm{O}_{2} \mathrm{O}_{2}+V_{2} V_{2} V_{2} V_{2}-C_{2} S_{2} S_{2} S_{2}-S_{2} C_{2} C_{2} C_{2} \\
& \tau_{\text {og }}^{(-)}=V_{2} V_{2} O_{2} O_{2}+O_{2} O_{2} V_{2} V_{2}-S_{2} C_{2} S_{2} S_{2}-C_{2} S_{2} C_{2} C_{2} \\
& \tau_{\text {oh }}^{(-)}=V_{2} O_{2} O_{2} V_{2}+O_{2} V_{2} V_{2} O_{2}-S_{2} S_{2} S_{2} C_{2}-C_{2} C_{2} C_{2} S_{2} \\
& \tau_{\text {of }}^{(-)}=V_{2} O_{2} V_{2} O_{2}+O_{2} V_{2} O_{2} V_{2}-S_{2} S_{2} C_{2} S_{2}-C_{2} C_{2} S_{2} C_{2} \\
& \tau_{\text {go }}^{(-)}=\mathrm{O}_{2} \mathrm{O}_{2} \mathrm{~S}_{2} C_{2}+V_{2} V_{2} C_{2} S_{2}-C_{2} S_{2} V_{2} O_{2}-S_{2} C_{2} O_{2} V_{2} \\
& \tau_{g g}^{(-)}=V_{2} V_{2} S_{2} C_{2}+O_{2} O_{2} C_{2} S_{2}-C_{2} S_{2} O_{2} V_{2}-S_{2} C_{2} V_{2} O_{2} \\
& \tau_{g h}^{(-)}=V_{2} O_{2} S_{2} S_{2}+O_{2} V_{2} C_{2} C_{2}-S_{2} S_{2} V_{2} V_{2}-C_{2} C_{2} O_{2} O_{2} \\
& \tau_{g f}^{(-)}=V_{2} O_{2} C_{2} C_{2}+O_{2} V_{2} S_{2} S_{2}-C_{2} C_{2} V_{2} V_{2}-S_{2} S_{2} O_{2} O_{2} \\
& \tau_{\text {ho }}^{(-)}=\mathrm{O}_{2} S_{2} C_{2} O_{2}+V_{2} C_{2} S_{2} V_{2}-S_{2} O_{2} V_{2} C_{2}-C_{2} V_{2} O_{2} S_{2}, \\
& \tau_{h g}^{(-)}=V_{2} C_{2} C_{2} O_{2}+O_{2} S_{2} S_{2} V_{2}-S_{2} O_{2} O_{2} S_{2}-C_{2} V_{2} V_{2} C_{2} \\
& \tau_{h h}^{(-)}=V_{2} S_{2} C_{2} V_{2}+O_{2} C_{2} S_{2} O_{2}-C_{2} O_{2} V_{2} S_{2}-S_{2} V_{2} O_{2} C_{2} \\
& \tau_{h f}^{(-)}=V_{2} S_{2} S_{2} O_{2}+O_{2} C_{2} C_{2} V_{2}-S_{2} V_{2} V_{2} S_{2}-C_{2} O_{2} O_{2} C_{2} \\
& \tau_{\text {fo }}^{(-)}=\mathrm{O}_{2} S_{2} \mathrm{O}_{2} C_{2}+V_{2} C_{2} V_{2} S_{2}-C_{2} V_{2} S_{2} O_{2}-S_{2} O_{2} C_{2} V_{2} \\
& \tau_{f g}^{(-)}=V_{2} C_{2} O_{2} C_{2}+O_{2} S_{2} V_{2} S_{2}-S_{2} O_{2} S_{2} O_{2}-C_{2} V_{2} C_{2} V_{2} \\
& \tau_{f h}^{(-)}=V_{2} S_{2} O_{2} S_{2}+O_{2} C_{2} V_{2} C_{2}-S_{2} V_{2} S_{2} V_{2}-C_{2} O_{2} C_{2} O_{2} \\
& \tau_{f f}^{(-)}=V_{2} S_{2} V_{2} C_{2}+O_{2} C_{2} O_{2} S_{2}-S_{2} V_{2} C_{2} O_{2}-C_{2} O_{2} S_{2} V_{2} .
\end{aligned}
$$




\section{B Characters for the $T^{6} / Z_{4}$ orbifold}

In this Appendix we list the characters needed for the $Z_{4}$ orientifold of Section 3:

$$
\begin{aligned}
& \rho_{00}=V_{2} O_{2} \chi_{0} \xi_{0}+O_{2} V_{2} \chi_{0} \xi_{4}-S_{2} C_{2} \chi_{0} \xi_{2}-C_{2} S_{2} \chi_{0} \xi_{-2}, \\
& \rho_{01}=V_{2} V_{2} \chi_{1 / 2} \xi_{-2}+O_{2} O_{2} \chi_{1 / 2} \xi_{2}-S_{2} S_{2} \chi_{1 / 2} \xi_{0}-C_{2} C_{2} \chi_{1 / 2} \xi_{4}, \\
& \rho_{02}=V_{2} O_{2} \chi_{0} \xi_{4}+O_{2} V_{2} \chi_{0} \xi_{0}-S_{2} C_{2} \chi_{0} \xi_{-2}-C_{2} S_{2} \chi_{0} \xi_{2}, \\
& \rho_{03}=V_{2} V_{2} \chi_{1 / 2} \xi_{2}+O_{2} O_{2} \chi_{1 / 2} \xi_{-2}-S_{2} S_{2} \chi_{1 / 2} \xi_{4}-C_{2} C_{2} \chi_{1 / 2} \xi_{0}, \\
& \rho_{10}=V_{2} S_{2} \chi_{0} \xi_{-3}+O_{2} C_{2} \chi_{0} \xi_{1}-S_{2} O_{2} \chi_{0} \xi_{-1}-C_{2} V_{2} \chi_{0} \xi_{3}, \\
& \rho_{11}=V_{2} C_{2} \chi_{1 / 2} \xi_{3}+O_{2} S_{2} \chi_{1 / 2} \xi_{-1}-S_{2} V_{2} \chi_{1 / 2} \xi_{-3}-C_{2} O_{2} \chi_{1 / 2} \xi_{1}, \\
& \rho_{12}=V_{2} S_{2} \chi_{0} \xi_{1}+O_{2} C_{2} \chi_{0} \xi_{-3}-S_{2} O_{2} \chi_{0} \xi_{3}-C_{2} V_{2} \chi_{0} \xi_{-1}, \\
& \rho_{13}=V_{2} C_{2} \chi_{1 / 2} \xi_{-1}+O_{2} S_{2} \chi_{1 / 2} \xi_{3}-S_{2} V_{2} \chi_{1 / 2} \xi_{1}-C_{2} O_{2} \chi_{1 / 2} \xi_{-3}, \\
& \rho_{20}=V_{2} O_{2} \chi_{1 / 2} \xi_{0}+O_{2} V_{2} \chi_{1 / 2} \xi_{4}-S_{2} C_{2} \chi_{1 / 2} \xi_{2}-C_{2} S_{2} \chi_{1 / 2} \xi_{-2}, \\
& \rho_{21}=V_{2} V_{2} \chi_{0} \xi_{-2}+O_{2} O_{2} \chi_{0} \xi_{2}-S_{2} S_{2} \chi_{0} \xi_{0}-C_{2} C_{2} \chi_{0} \xi_{4}, \\
& \rho_{22}=V_{2} O_{2} \chi_{1 / 2} \xi_{4}+O_{2} V_{2} \chi_{1 / 2} \xi_{0}-S_{2} C_{2} \chi_{1 / 2} \xi_{-2}-C_{2} S_{2} \chi_{1 / 2} \xi_{2}, \\
& \rho_{23}=V_{2} V_{2} \chi_{0} \xi_{2}+O_{2} O_{2} \chi_{0} \xi_{-2}-S_{2} S_{2} \chi_{0} \xi_{4}-C_{2} C_{2} \chi_{0} \xi_{0}, \\
& \rho_{30}=V_{2} C_{2} \chi_{0} \xi_{3}+O_{2} S_{2} \chi_{0} \xi_{-1}-S_{2} V_{2} \chi_{0} \xi_{-3}-C_{2} O_{2} \chi_{0} \xi_{1}, \\
& \rho_{31}=V_{2} S_{2} \chi_{1 / 2} \xi_{1}+O_{2} C_{2} \chi_{1 / 2} \xi_{-3}-S_{2} O_{2} \chi_{1 / 2} \xi_{3}-C_{2} V_{2} \chi_{1 / 2} \xi_{-1}, \\
& \rho_{32}=V_{2} C_{2} \chi_{0} \xi_{-1}+O_{2} S_{2} \chi_{0} \xi_{3}-S_{2} V_{2} \chi_{0} \xi_{1}-C_{2} O_{2} \chi_{0} \xi_{-3}, \\
& \rho_{33}=V_{2} S_{2} \chi_{1 / 2} \xi_{-3}+O_{2} C_{2} \chi_{1 / 2} \xi_{1}-S_{2} O_{2} \chi_{1 / 2} \xi_{-1}-C_{2} V_{2} \chi_{1 / 2} \xi_{3} .
\end{aligned}
$$

The $\sigma$ characters are obtained interchanging in the internal part $O_{2}$ with $V_{2}, \xi_{0}$ with $\xi_{4}, S_{2}$ with $C_{2}$, and $\xi_{2}$ with $\xi_{-2}$ :

$$
\sigma_{00}=V_{2} V_{2} \chi_{0} \xi_{4}+O_{2} O_{2} \chi_{0} \xi_{0}-S_{2} S_{2} \chi_{0} \xi_{-2}-C_{2} C_{2} \chi_{0} \xi_{2}
$$




$$
\begin{aligned}
& \sigma_{01}=V_{2} O_{2} \chi_{1 / 2} \xi_{2}+O_{2} V_{2} \chi_{1 / 2} \xi_{-2}-S_{2} C_{2} \chi_{1 / 2} \xi_{4}-C_{2} S_{2} \chi_{1 / 2} \xi_{0} \\
& \sigma_{02}=V_{2} V_{2} \chi_{0} \xi_{0}+O_{2} O_{2} \chi_{0} \xi_{4}-S_{2} S_{2} \chi_{0} \xi_{2}-C_{2} C_{2} \chi_{0} \xi_{-2}, \\
& \sigma_{03}=V_{2} O_{2} \chi_{1 / 2} \xi_{-2}+O_{2} V_{2} \chi_{1 / 2} \xi_{2}-S_{2} C_{2} \chi_{1 / 2} \xi_{0}-C_{2} S_{2} \chi_{1 / 2} \xi_{4} \\
& \sigma_{20}=O_{2} O_{2} \chi_{1 / 2} \xi_{0}+V_{2} V_{2} \chi_{1 / 2} \xi_{4}-S_{2} S_{2} \chi_{1 / 2} \xi_{-2}-C_{2} C_{2} \chi_{1 / 2} \xi_{2} \\
& \sigma_{21}=V_{2} O_{2} \chi_{0} \xi_{-2}+O_{2} V_{2} \chi_{0} \xi_{2}-S_{2} C_{2} \chi_{0} \xi_{0}-C_{2} S_{2} \chi_{0} \xi_{4}, \\
& \sigma_{22}=V_{2} V_{2} \chi_{1 / 2} \xi_{0}+O_{2} O_{2} \chi_{1 / 2} \xi_{4}-S_{2} S_{2} \chi_{1 / 2} \xi_{2}-C_{2} C_{2} \chi_{1 / 2} \xi_{-2} \\
& \sigma_{23}=V_{2} O_{2} \chi_{0} \xi_{2}+O_{2} V_{2} \chi_{0} \xi_{-2}-S_{2} C_{2} \chi_{0} \xi_{4}-C_{2} S_{2} \chi_{0} \xi_{0} .
\end{aligned}
$$




\section{References}

[1] A. Sagnotti, in: Cargese '87, Non-Perturbative Quantum Field Theory, eds. G. Mack et al. (Pergamon Press, Oxford, 1988) p. 521; G. Pradisi and A. Sagnotti, Phys. Lett. B216 (1989) 59; M. Bianchi and A. Sagnotti, Phys. Lett. B247 (1990) 517, Nucl. Phys. B361 (1991) 519.

[2] I. Antoniadis, Phys. Lett. B246 (1990) 377.

[3] E. Witten, Nucl. Phys. B471 (1996) 135; J.D. Lykken, Phys. Rev. D54 (1996) 3693.

[4] N. Arkani-Hamed, S. Dimopoulos and G. Dvali, Phys. Lett. B429 (1998) 263; K.R. Dienes, E. Dudas and T. Gherghetta, Phys. Lett. B436 (1998) 55; I. Antoniadis, N. Arkani-Hamed, S. Dimopoulos and G. Dvali, Phys. Lett. B436 (1998) 263; for a recent review see I. Antoniadis, hep-th/9909212 and references therein.

[5] K. Benakli, Phys. Rev. D60 (1999) 104002; C. Burgess, L.E. Ibáñez and F. Quevedo, Phys. Lett. B447 (1999) 257.

[6] J. Polchinski and Y.C. Cai, Nucl. Phys. B296 (1988) 91.

[7] J. Polchinski, Phys. Rev. Lett. 75 (1995) 4724.

[8] M. Bianchi, Ph.D. Thesis, preprint ROM2F-92/13; A. Sagnotti, hep-th/9302099.

[9] G. Zwart, Nucl. Phys. B526 (1998) 378; Z. Kakushadze, G. Shiu and S.H.H. Tye, Nucl. Phys. B533 (1998) 25; G. Aldazabal, A. Font, L.E. Ibáñez and G. Violero, Nucl. Phys. B536 (1998) 29.

[10] I. Antoniadis, E. Dudas and A. Sagnotti, Phys. Lett. B464 (1999) 38.

[11] R. Rohm, Nucl. Phys. B237 (1984) 553; C. Kounnas and M. Porrati, Nucl. Phys. B310 (1988) 355; I. Antoniadis, C. Bachas, D.C. Lewellen and T.N. Tomaras, Phys. Lett. 
B207 (1988) 441; S. Ferrara, C. Kounnas, M. Porrati and F. Zwirner, Nucl. Phys. B318 (1989) 75; C. Kounnas and B. Rostand, Nucl. Phys. B341 (1990) 641; I. Antoniadis, Phys. Lett. B246 (1990) 377; I. Antoniadis and C. Kounnas, Phys. Lett. B261 (1991) 369 .

[12] I. Antoniadis, E. Dudas and A. Sagnotti, Nucl. Phys. B544 (1999) 469; I. Antoniadis, G. D’Appollonio, E. Dudas and A. Sagnotti, Nucl. Phys. B553 (1999) 133.

[13] R. Blumenhagen and L. Görlich, Nucl. Phys. B551 (1999) 601; C. Angelantonj, I. Antoniadis and K. Förger, Nucl. Phys. B555 (1999) 116.

[14] I. Antoniadis, G. D'Appollonio, E. Dudas and A. Sagnotti, hep-th/9907184, to appear in Nucl. Phys. B; A.L. Cotrone, hep-th/9909116.

[15] C. Bachas, hep-th/9503030; M. Bianchi and Ya.S. Stanev, Nucl. Phys. B253 (1998) 193.

[16] M. Bianchi, J.F. Morales and G. Pradisi, hep-th/9910228.

[17] S. Sugimoto, hep-th/9905159.

[18] G. Aldazabal and A.M. Uranga, hep-th/9908072.

[19] C. Angelantonj, M. Bianchi, G. Pradisi, A. Sagnotti and Ya.S. Stanev, Phys. Lett. B385 (1996) 96.

[20] G. Aldazabal, L.E. Ibáñez and F. Quevedo, hep-th/9909172.

[21] A. Sen, J. High Energy Phys. 9806 (1998) 007, 9808 (1998) 010, 012, 9809 (1998) 023, 9812 (1998) 021. For recent reviews, see A. Sen, hep-th/9904207; A. Lerda and R. Russo, hep-th/9905006.

[22] C. Vafa, Nucl. Phys. B273 (1986) 592; C. Vafa and E. Witten, J. Geom. Phys. 15 (1995) 189; J. Blum, Nucl. Phys. B486 (1997) 34; M.R. Douglas, hep-th/9807235. 
[23] D. Fioravanti, G. Pradisi and A. Sagnotti, Phys. Lett. B321 (1994) 349; G. Pradisi, A. Sagnotti and Ya.S. Stanev, Phys. Lett. B354 (1995) 279, B356 (1995) 230, B381 (1996) 97.

[24] M. Berkooz and R.G. Leigh, Nucl. Phys. B483 (1997) 187.

[25] Z. Kakushadze, G. Shiu and S.H.H. Tye, in [9]; K. Mohri, Nucl. Phys. B521 (1998) 161; S. Mukhopadhyay and K. Ray, hep-th/9909107.

[26] M. Bianchi, G. Pradisi and A. Sagnotti, Nucl. Phys. B376 (1992) 365

[27] M.B. Green and J.H. Schwarz, Phys. Lett. B149 (1984) 117.

[28] Z. Kakushadze, G. Shiu and S.H.H. Tye, Phys. Rev. D58 (1998) 086001; C. Angelantonj, hep-th/9908064, to appear in Nucl. Phys. B.

[29] A. Sagnotti, hep-th/9509080, hep-th/9702093, C. Angelantonj, Phys. Lett. B444 (1998) 309; R. Blumenhagen, A. Font and D. Lüst, hep-th/9904069; R. Blumenhagen and A. Kumar, hep-th/9906234; K. Förger, hep-th/9909010.

[30] M. Bianchi and A. Sagnotti, in [1].

[31] E. Gimon and J. Polchinski, hep-th/9601038.

[32] A. Sagnotti, Phys. Lett. B294 (1992) 196.

[33] J. Blum and K.R. Dienes, Phys. Lett. B414 (1997) 260, Nucl. Phys. B516 (1998) 83.

[34] R. Blumenhagen, C. Kounnas and D. Lüst, hep-th/9910094.

[35] I. Antoniadis and M. Quiros, Phys. Lett. B392 (1997) 61; E. Dudas and C. Grojean, Nucl. Phys. B507 (1997) 553, hep-th/9704177; I. Antoniadis and M. Quiros, Nucl. Phys. B505 (1997) 109, hep-th/9705037, Phys. Lett. B416 (1998) 327; E. Dudas, Phys. Lett. B416 (1998) 309. 